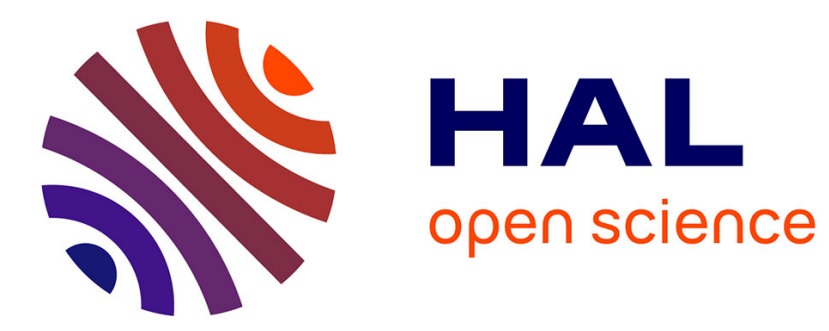

\title{
Microanalyse et microscopie photoélectroniques X: principe et performances prévisibles
}

\author{
J. Cazaux
}

\section{To cite this version:}

J. Cazaux. Microanalyse et microscopie photoélectroniques X: principe et performances prévisibles. Revue de Physique Appliquée, 1975, 10 (5), pp.263-280. 10.1051/rphysap:01975001005026300 . jpa00243912

\section{HAL Id: jpa-00243912 https://hal.science/jpa-00243912}

Submitted on 1 Jan 1975

HAL is a multi-disciplinary open access archive for the deposit and dissemination of scientific research documents, whether they are published or not. The documents may come from teaching and research institutions in France or abroad, or from public or private research centers.
L'archive ouverte pluridisciplinaire HAL, est destinée au dépôt et à la diffusion de documents scientifiques de niveau recherche, publiés ou non, émanant des établissements d'enseignement et de recherche français ou étrangers, des laboratoires publics ou privés. 


\begin{abstract}
Classification
Physics Abstracts

$0.691-0.695-8.960$

\section{MICROANALYSE ET MICROSCOPIE PHOTOÉLECTRONIQUES X : PRINCIPE ET PERFORMANCES PRÉVISIBLES (*)}

\author{
J. CAZAUX \\ Groupe de spectroscopie des électrons $(* *)$ \\ Faculté des Sciences, 51062 Reims Cedex, France
}

(Reçu le 24 février 1975, accepté le 29 avril 1975)

\begin{abstract}
Résumé. - En calculant l'intensité des photoélectrons $X$ émis par un objet ayant la forme d'un film mince et placé sous une anticathode à faces parallèles, nous montrons qu'il est possible de conférer à la spectroscopie des photoélectrons un caractère microanalytique avec une résolution spatiale de l'ordre de $1 \mu$.

Nous en déduisons, pour le microscope à balayage, un mode de fonctionnement avec lequel on obtiendrait une image spécifique non seulement de la nature des éléments constituant la préparation mais aussi de leur degré d'oxydation. Après les avoir comparés, nous indiquons comment l'appareil projeté permet de combiner les mesures effectuées en spectrométries Auger et en ESCA.
\end{abstract}

\begin{abstract}
The $X$ ray photoelectron intensity issued from a thin film sample attached below an anode (in a sheet form) is calculated and the principles and expected performances of photoelectron microanalysis are deduced.

In scanning electron microscopy, using the proposed operating conditions, it will be possible to obtain a final electronic image not only characteristic of a given element but also of its oxydation degree with a spatial resolution of about $1 \mu$. A.E.S. and X.P.S. are compared and the schematic drawing of a versatile apparatus allowing electron induced A.E.S., $X$ ray induced A.E.S. and X.P.S. is pointed out.
\end{abstract}

1. Introduction. - Nous avons assisté au cours des dernières décades à la conception et au développement de nombreuses techniques de microanalyse : microsonde électronique, spectroscopie de masse, etc... dont le principe repose sur l'analyse de rayonnements caractéristiques secondaires (photons $\mathrm{X}$, électrons ou ions) émis par une cible solide sous l'impact de faisceaux primaires (électrons, ions ou photons $\mathrm{X}$ ), afin de déterminer les éléments constituant celle-ci.

Parmi ces techniques, la spectroscopie des photoélectrons (excités par une radiation $\mathrm{X}$ monochromatique) occupe une place particulière dans la mesure où elle permet non seulement de déterminer la nature des éléments qui constituent la surface de la préparation observée mais aussi leur degré d'oxydation (glissement chimique). Mais si la faible profondeur d'extraction $\lambda_{\mathrm{e}}$ des photoélectrons ( $\lambda_{\mathrm{e}}$ est de l'ordre de $10 \AA$ ) confère à

(*) Les résultats préliminaires relatifs à ce travail ont été présentés au $8^{\mathrm{e}}$ Congrès International de Microscopie Electronique de CANBERRA I (1974), p. 72.

$(* *)$ Laboratoire de Microscopie et de Spectroscopie Electroniques E. R. A. 376. cette technique un caractère d'étude des surfaces, elle nécessite en contrepartie que l'aire analysée soit assez étendue pour que le signal reçu puisse être détecté : c'est ainsi que, dans la plupart des spectromètres photoélectroniques, cette aire atteint plusieurs millimètres carrés et interdit ainsi à cette technique d'être vraiment microanalytique.

Pourtant, comme l'amélioration de la résolution spatiale de ces instruments présente un intérêt évident, nous nous proposons de montrer qu'en plaçant l'objet (sous forme de film mince) le plus près possible de la source $\mathrm{X}$, on peut réduire l'aire analysée à quelques microns carrés et mieux.

Il sera alors possible, comme dans la plupart des dispositifs de microanalyse ci-dessus mentionnés, de former à partir d'une radiation caractéristique - ici une raie photoélectronique - une image à 2 dimensions donnant la distribution spatiale d'un élément dans un état d'oxydation particulier.

Nous décrirons donc dans une première partie le principe et la conception générale du dispositif expérimental, puis ( $2^{\mathrm{e}}$ partie) nous calculerons l'intensité photoélectronique dans ces conditions expérimentales 
et nous déterminerons les valeurs à donner aux différents paramètres ajustables pour optimiser ce flux.

Nous évaluerons ensuite la résolution spatiale du dispositif proposé et enfin en guise de conclusion (4e partie) nous comparerons les performances des appareils préconisés avec celles d'autres instruments susceptibles de fournir des informations comparables sur les surfaces (appareils reposant essentiellement sur l'effet Auger).

2. Principe et conception générale de l'appareil. 2.1 PRINCIPE. - Les caractéristiques de la spectroscopie des photoélectrons sont bien connues (voir par exemple les travaux de Siegbahn et coll. [1] ou les comptes rendus des congrès spécialisés dans cette technique, Asilomar [2], Namur [3]), aussi dans les multiples aspects de cette discipline nous ne retiendrons aux fins de microanalyse que les points suivants:

- le spectre photoélectronique d'un solide excité par une radiation $\mathrm{X}$ monochromatique $(h v)$ se présente sous forme de raies et de bandes dont la position énergétique (énergie cinétique) reflète $\left(h v=E_{\mathrm{C}}+E_{\mathrm{L}}\right)$ l'énergie de liaison des états électroniques $\left(E_{\mathrm{L}}\right)$ du solide :

- par opposition à la position énergétique des bandes (relatives aux bandes de valence et de conduction $E_{\mathrm{L}}<20 \mathrm{eV}$ ), la position des raies du spectre (relative à l'excitation des niveaux électroniques profonds $E_{\mathrm{L}}>20 \mathrm{eV}$ ) est en première approximation caractéristique des éléments qui constituent le solide étudié ;

- de façon plus précise la position des raies d'un élément donné est sensible aussi à la nature des liaisons existant entre un atome considéré et les atomes environnants (glissement chimique) et l'amplitude de cette perturbation peut atteindre une dizaine d'électronsvolts.

On trouvera dans la littérature déjà citée des exemples abondants concernant cet effet (voir par exemple le Tableau I dans [4]).

La largeur énergétique des raies considérées est fonction des 3 paramètres suivants : la largeur énergétique du niveau atomique considéré, la largeur naturelle de la radiation $X$ incidente et la bande passante instrumentale liée au pouvoir de résolution de l'analyseur de vitesse électronique ; par un choix judicieux de la radiation $\mathrm{X}$ excitatrice et avec un spectromètre correctement construit, l'ordre de grandeur de cette largeur globale (1 eV environ) permet sans difficulté de mettre en évidence le glissement chimique ci-dessus mentionné.

Notons enfin que la spectroscopie des photoélectrons $\mathrm{X}$ est un phénomène superficiel dans la mesure où la profondeur d'extraction $\lambda_{\mathrm{e}}$ des électrons excède rarement $30 \AA$.

C'est ainsi que la spectroscopie des photoélectrons $\mathrm{X}$ fournit des spectres électroniques non seulement caractéristiques des éléments qui constituent la couche superficielle de la préparation mais aussi de leur environnement chimique.

2.2 CONCEPTION GÉNÉRALE (microanalyse et microscopie photoélectroniques X). - Dans les spectrographes conventionnels la source $X$ (anticathode) est distante de l'objet d'une distance de l'ordre de $10 \mathrm{~cm}$ et, dans ces conditions, l'intensité d'une raie caractéristique est de l'ordre de $10^{4}$ à $10^{5} \mathrm{cps} / \mathrm{s}$ pour une aire éclairée ayant les dimensions latérales dépassant le millimètre.

Il est évident qu'en maintenant constant l'angle solide sous lequel de la source on voit l'objet - c'est-à-dire en conservant la même intensité $X$ sur la préparation : on réduira les dimensions latérales proportionnellement à la réduction de la distance entre la source et l'objet.

A partir de sources $\mathrm{X}$ conventionnelles, il est donc clair que la microanalyse photoélectronique ne peut être réalisée que pour la situation extrême dans laquelle l'objet est placé très près de l'anticathode.

On peut à la limite, concevoir (Ichikawa et Tochigi [5], Citrin et coll. [6], Cazaux [4]) que cet objet ayant la forme d'un film mince soit placé sous celle-ci :

Les électrons incidents (Fig. 1a) sont focalisés sur la partie supérieure d'une anticathode ayant la forme d'une lame à faces parallèles, les photons $\mathrm{X}$ émis se propagent à travers celle-ci et à travers l'objet pour éclairer la face inférieure de l'échantillon, les photoélectrons émis sont éjectés dans le vide et analysés par un spectrographe ayant un coefficient de transmission élevé.

$\mathrm{Si}$ on peut conférer à la source photoélectronique des dimensions réduites (quelques microns ou mieux) tout en conservant une émission suffisamment intense pour être détectée, on pourra, en appliquant une tension variable sur l'analyseur électrostatique, obtenir le spectre de photoélectrons relatif à la zone émissive et ainsi réaliser une microanalyse locale de l'échantillon.

Le spectrographe étant calé sur une raie photoélectronique déterminée, il sera possible en outre, par simple balayage de l'anticathode avec le faisceau électronique incident et à l'aide de la détection synchrone des signaux sortant de l'analyseur, de restituer sur un écran de télévision l'image obtenue avec les photoélectrons relatifs à cette raie (c'est-à-dire issus d'un élément donné dans un état d'oxydation prédéterminé).

Le montage correspondant est schématisé sur la figure $1 b$ avec, à titre d'exemple, un analyseur du type miroir cylindrique ; le passage d'un mode de fonctionnement à l'autre pourra s'effectuer par de simples commutations électroniques.

Remarques. - 1) L'obtention globale d'une image photoélectronique filtrée a été initialement proposée par l'auteur [4] mais il fallait alors, pour obtenir une résolution spatiale acceptable, utiliser des électrons dont l'énergie cinétique était inférieure à $40 \mathrm{eV}$; outre 

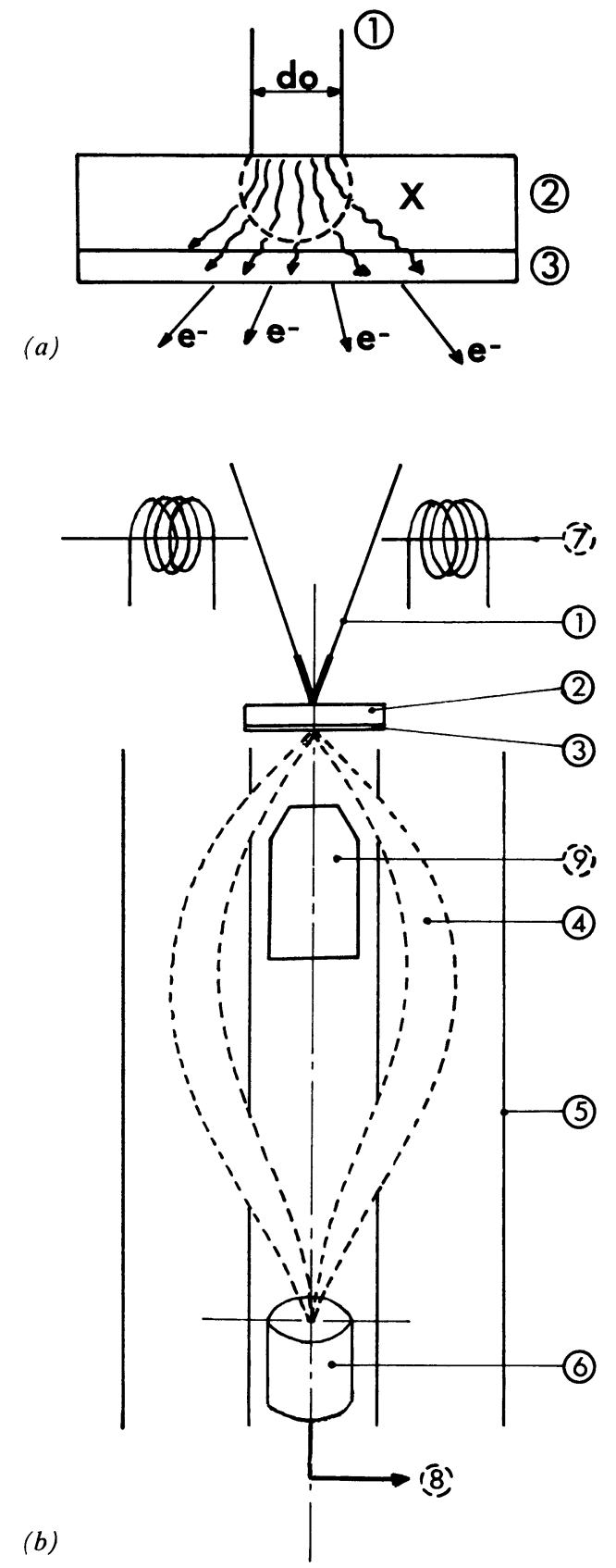

FIG. 1. - a) Système anticathode objet. b) Schéma de principe du microanalyseur et du microscope photoélectronique $\mathbf{X}$. Microanalyseur: (1) Sonde électronique. (2) Anticathode. (3) Objet. (4) Faisceau électronique. (5) Analyseur miroir cylindrique (par exemple). (6) Détecteur. Microscope photoélectronique à balayage: en plus. (7) Bobines de balayage. (8) Vers le système de télévision. Accessoire supplémentaire pour l'analyse Auger : (9) Canon à électrons.

l'inconvénient introduit par l'emploi de plusieurs types d'anticathodes, il semble que nous ayons sous-estimé l'influence de divers processus (électrons secondaires, processus inélastiques multiples) qui tendent à produire un fond continu très intense d'où émergent à peine les raies relatives à ces électrons de faible énergie cinétique. Par contre, la reconstruction point par point de l'image, n'imposant plus de conditions sur l'énergie cinétique des électrons, pourra s'effectuer sans pro- blème à partir des raies photoélectriques qui émergent nettement du fond continu.

2) Pour estimer ultérieurement l'ordre de grandeur des intensités photoélectroniques reçues par le détecteur, nous supposerons que l'analyseur est du type miroir cylindrique (Fig. 1b) et que ses dimensions correspondent à celui mis au point par Citrin et coll. [6].

Toutefois l'emploi de tout autre type d'analyseur est bien sûr concevable comme par exemple l'analyseur à grilles hémisphériques de Staib [7] qui présente l'intérêt, vis-à-vis d'autres types d'appareils, d'avoir une acceptance angulaire comparable aux analyseurs à champ retardateur habituellement employés en diffraction d'électrons lents $\left(\Delta \theta \simeq 20^{\circ}\right)$ tout en fonctionnant en filtre passe bande.

3. Intensité photoélectronique et optimisation des paramètres. - 3.1 INTRODUCTION. - Dans ce chapitre nous nous proposons d'évaluer, dans la géométrie indiquée ci-dessus (Fig. 1a), l'ordre de grandeur de l'intensité photoélectronique pénétrant dans l'analyseur et correspondant à la détection d'une raie bien déterminée émise par l'échantillon.

- Pour simplifier les résultats et faciliter leur discussion nous effectuons cette estimation en considérant que la source $X$ est ponctuelle vis-à-vis de l'objet; aussi dans le paragraphe 3.2 nous cherchons d'abord à établir quel diamètre « $d_{0}$ » il faut donner à la sonde électronique incidente pour que cette approximation soit justifiée : nous obtenons ainsi l'inégalité (4) par l'intermédiaire des relations (1), (2) et (3).

- Dans le paragraphe 3.3, nous établissons l'expression littérale (11) donnant le nombre de photoélectrons monocinétiques pénétrant par seconde dans l'analyseur soit $N(\theta, \Delta \theta)$ en fonction du nombre de photons émis (par seconde) par la source $\mathrm{X}$ (soit $Q$ ) et de la distance « $h$ » de la source $\mathrm{X}$ à l'objet.

- Comme dans le problème de la microsonde de Castaing [8], pour optimiser cette intensité photoélectronique émise, il faut optimiser $Q$ par un choix judicieux des valeurs à donner aux paramètres ajustables tels que la tension $\left(V_{0}\right)$, l'intensité $\left(I_{0}\right)$ et le diamètre de la sonde électronique $\left(d_{0}\right)$ avec de plus, dans notre cas, l'épaisseur de l'anticathode (dont la nature a été préétablie). Nous traitons ces différents aspects dans le paragraphe 3.4 et nous montrons que, compte tenu des limites physiques imposées par l'émission thermoionique (16) ou par l'échauffement de l'anticathode (17), ces paramètres ajustables sont interdépendants et que leur valeur optimale peut s'exprimer en fonction de $V_{0}$ (tension d'accélération des électrons incidents).

- Enfin dans le paragraphe 3.5, nous estimons numériquement, pour fixer les idées, l'ordre de grandeur du nombre de photoélectrons détectés par seconde et correspondant à une raie caractéristique de l'or (objet) pour différentes valeurs de la tension $V_{0}$ et pour des anticathodes en aluminium et niobium. 
Les résultats obtenus sont illustrés dans la figure 6 et les conditions expérimentales sont précisées dans les tableaux I, II et III.

\subsection{Modèle DE Diffusion DANS L'ANTICATHOdE ET} DIAMÈTRE DE LA SONDE INCIDENTE. - L'ensemble des trajectoires des électrons incidents dans l'anticathode définissent sensiblement un volume de révolution dans lequel les rayons $X$ sont créés; nous allons chercher à évaluer le diamètre maximal à donner à la sonde électronique pour qu'en première approximation la source $\mathrm{X}$ correspondante puisse être considérée comme ponctuelle vis-à-vis de l'objet situé sous l'anticathode. Pour faire cette évaluation, nous négligerons pour l'instant l'absorption de rayons $\mathrm{X}$ dans l'anticathode (nous en tiendrons compte ultérieurement au § 3.3) et nous supposerons que les électrons incidents suivent le modèle de diffusion d'Archard [9] car on ne peut ici, par opposition à la microanalyse par sonde électronique, envisager l'emploi de la fonction $(\rho z)$ qui, bien accessible à l'expérience (Castaing et Deschamps [11]) et pouvant être approchée par la théorie (Philibert [10]), ne permet pas de tenir compte simplement de l'influence du diamètre de la sonde incidente.

Nous admettrons donc (voir Fig. 2a) que l'électron incident pénètre de façon rectiligne dans l'anticathode jusqu'à la profondeur « $z_{\mathrm{D}}$ " de complète diffusion puis est diffusé isotropiquement jusqu'à ce que son parcours total soit égal à la profondeur de pénétration $" z_{\mathbf{M}}$ 》.

Si l'anticathode est bombardée par un faisceau cylindrique homogène d'électrons de diamètre $d_{0}$, on peut admettre que la zone de production des rayons $\mathrm{X}$ est assimilable à un ellipsoïde de révolution autour de l'axe du faisceau électronique incident et ayant pour demi-grand axe

$$
a=\frac{d_{0}}{2}+\mathrm{z}_{\mathrm{M}}-\mathrm{z}_{\mathrm{D}}
$$

(voir Fig. $2 b$ ) et pour demi-petit axe $c=Z_{M}-Z_{\mathrm{D}}$. Or le flux de photons $\mathrm{d}^{2} \Phi$ émis par un élément de volume $\mathrm{d} v$ (centré en $P$ ) à travers une surface $\mathrm{d} S$ (centrée en $M$ ) est tel que (en supposant une émission isotrope et sans atténuation) :

$$
\mathrm{d}^{2} \Phi=\frac{\sigma \mathrm{d} v}{4 \pi} \frac{\mathrm{d} S \cos \alpha}{\overline{P M}^{2}}=\frac{\sigma \mathrm{d} v}{4 \pi} \frac{\mathrm{d} \mathbf{S r}}{r^{3}}
$$

où $\sigma$ représente la densité de photons $\mathrm{X}$ émis par unité de volume et $\mathbf{r}=\mathbf{P M}$. On observera que ce flux est identique (à $\varepsilon_{0}$ près dans le système MKSA) au flux, dans les mêmes conditions géométriques, du champ électrique créé par une distribution volumique de charges $\sigma$ à travers la même surface $\mathrm{d} \mathbf{S}$; or le problème du champ électrique $\mathbf{E}$ créé par un ellipsoïde uniformément chargé $\sigma$ a été résolu littéralement par Durand [12] : en suivant les notations de cet auteur, nous pouvons calculer aisément le nombre de photons $\mathrm{X}$ [soit $\left.I X\left(R_{0}\right)\right]$ traversant par seconde la circonférence de rayon $R_{0}$ perpendiculaire à l'axe de

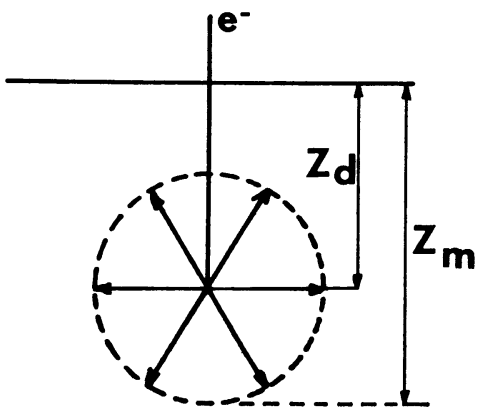

(a)
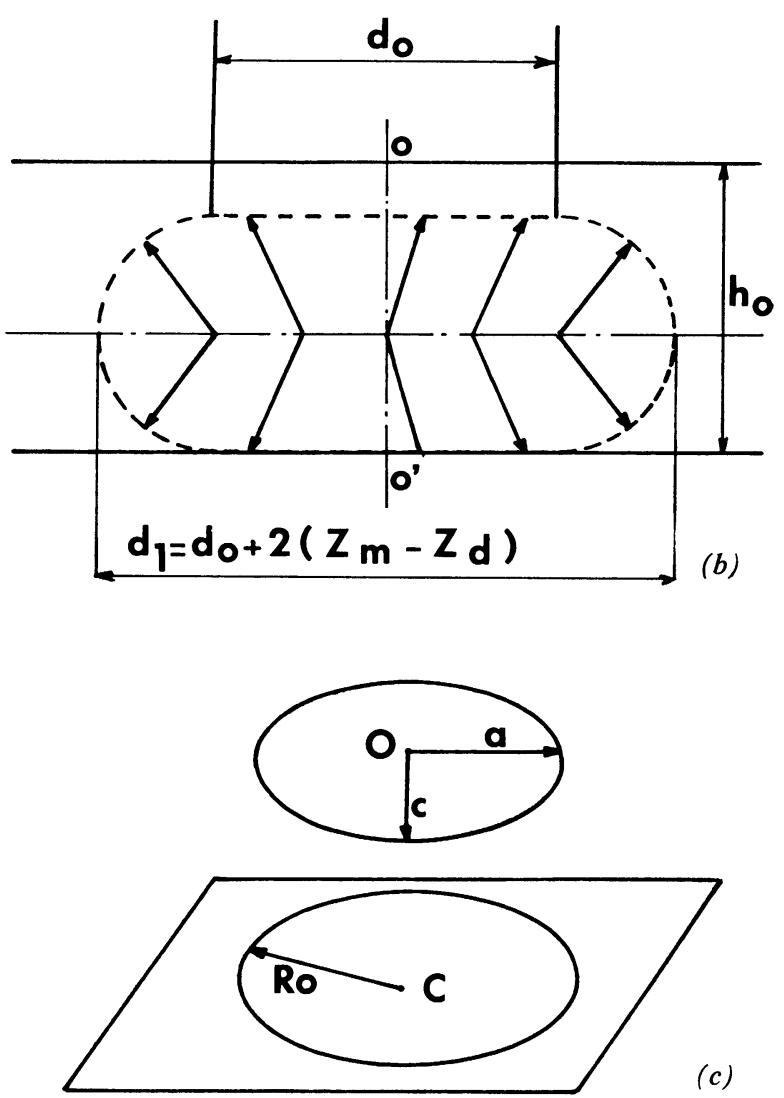

Fig. 2. - Modèle de diffusion d'Archard. a) Cas d'un seul électron incident. b) Hypothèse d'un faisceau cylindrique incident. c) Assimilation de la zone de création des rayons $\mathrm{X}$ à un ellipsoïde de révolution.

révolution de l'ellipsoïde et centrée en un point $c$ distant de $« h »$ du centre $O$ de cet ellipsoïde (Fig. $2 c$ )

$$
\begin{aligned}
I X\left(R_{0}\right) & =\int_{0}^{R_{0}} \varepsilon_{0} E_{L} \cdot 2 \pi R \mathrm{~d} R \\
& =\pi a^{2} c \sigma h \int_{0}^{R_{0}} R \mathrm{~d} R \int_{\xi}^{\infty} \frac{\mathrm{d} \lambda}{\left(c^{2}+\lambda\right)^{3 / 2}\left(a^{2}+\lambda\right)}
\end{aligned}
$$

avec $\xi>0$ tel que

$$
\frac{R_{0}^{2}}{a^{2}+\xi}+\frac{h^{2}}{c^{2}+\xi}=1,
$$

ce qui conduit au résultat suivant (quand on prend $h=c)$ : 


$$
\begin{array}{r}
I X\left(R_{0}\right)=\frac{Q}{2}\left[\frac{3\left(X^{2}-1\right)}{2 e^{2} X}-\frac{3\left(X^{2}-1\right)\left(e^{2}+X^{2}\right)}{2 e^{3} X^{2}} \times\right. \\
\left.\times \operatorname{tg}^{-1}\left(\frac{e}{X}\right)-\frac{1}{X^{3}}\right]_{1}^{X_{0}}
\end{array}
$$

avec $X_{0}$ tel que :

$$
\frac{R_{0}^{2} / c^{2}}{X_{0}^{2}+e^{2}}+\frac{1}{X_{0}^{2}}=1,
$$

l'excentricité « $e$ » de l'ellipsoïde étant égale à :

$$
\frac{1}{c} \sqrt{a^{2}-c^{2}}
$$

et $Q$ le nombre de photons $\mathrm{X}$ émis par seconde.

A partir de l'expression (3), nous avons représenté sur la figure 3 l'évolution de $I X$ en fonction de $R_{0}$ pour différentes valeurs de l'excentricité ; on vérifie bien que, quand $R \rightarrow \infty$, la moitié de l'intensité $X$ émise par la source (soit $Q / 2$ ) traverse le plan inférieur et que, d'autre part, l'intensité traversant une circonférence de rayon $R \simeq 2 c$ ou $3 c$ est d'autant plus faible que l'excentricité est grande et donc que le faisceau électronique incident a un diamètre élevé.

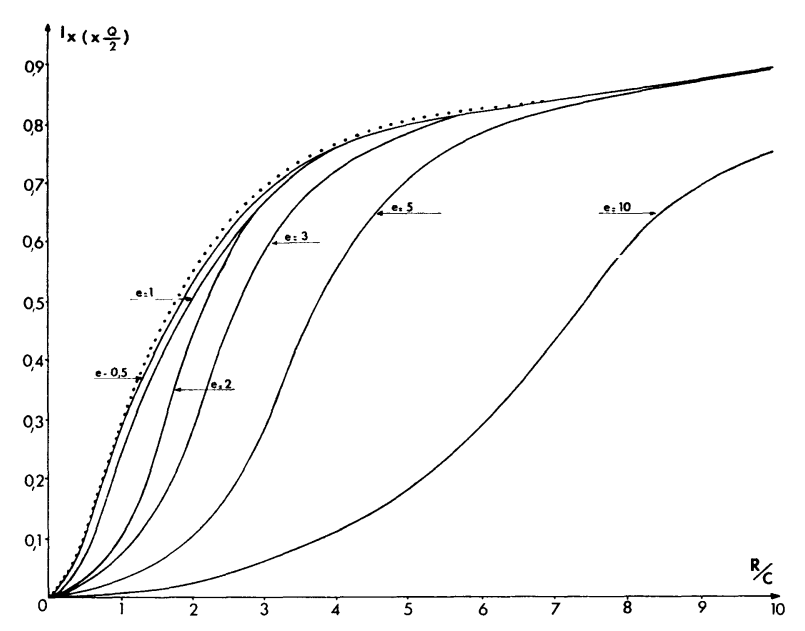

FIG. 3. - Evolution de l'intensité $I(\mathrm{X})$ traversant une circonférence de rayon $R_{0}$ en fonction de différentes valeurs de l'ellipticité de la source (La courbe en pointillés représente le cas d'une source ponctuelle.) (L'atténuation des X n'est pas prise en compte.)

Nous pouvons toutefois constater que, tant que l'excentricité n'excède pas $e \simeq 2$ ou 3 , la répartition de l'intensité $X$ dans le plan objet s'écarte assez peu de celle qui serait donnée par une source $X$ ponctuelle placée au centre $O$ de l'ellipsoïde (courbe en pointillé Fig. 3).

Pour des raisons de simplicité, nous calculerons donc par la suite l'intensité photoélectronique en tenant compte de l'auto-absorption du rayonnement $X$ dans l'anticathode mais en supposant que la source $X$ est ponctuelle, c'est-à-dire en prenant :

$$
e \leqslant 3 \quad \text { soit } \quad d_{0} \lesssim 4 c \simeq 4\left(z_{M}-z_{\mathrm{D}}\right) .
$$

Cette source ponctuelle devrait en principe être placée à la distance minimale $h=c=Z_{m}-Z_{d}$ au-dessous du plan contenant l'échantillon (pour minimiser l'autoabsorption du rayonnement $X$ et surtout - voir plus loin - optimiser la résolution spatiale) mais un tel choix, prenant trop à la lettre le modèle d'Archard, négligerait le fait que, statistiquement, certains électrons incidents pouvant traverser anticathode et objet viendraient se mêler aux photoélectrons pour créer, dans le spectre final, un fond continu nuisible (quand l'énergie cinétique de ces électrons parasites coïncidera avec l'énergie cinétique d'une raie photoélectronique). Pour prévenir cette éventualité, nous placerons (Fig. 4) la source ponctuelle à une distance $h$ supérieure à « $c$ » et les applications numériques ultérieures correspondent à $h=2 c$ en incluant l'épaisseur de l'objet (voir plus loin). L'épaisseur de l'anticathode obéira, donc, à la relation :

$$
t_{1}=z_{\mathrm{D}}+h=2 z_{\mathrm{M}}-z_{\mathrm{D}} .
$$

3.3 EXPRESSION DONNANT L'INTENSITÉ PHOTOÉLECTRONIQUE ÉMISE. - L'évaluation théorique de l'intensité photoélectronique traversant la fente d'entrée d'un analyseur a été effectuée par Henke [13] puis par Fadley et coll. [14] à partir de la géométrie usuelle des spectromètres à photoélectrons.

Nous allons reprendre leur démarche en l'adaptant à la géométrie particulière que nous préconisons ici. Nous allons, comme eux, faire les hypothèses simplificatives suivantes.

a) L'échantillon est homogène et a la forme d'une lame à faces parallèles d'épaisseur $t_{0}$.

b) Nous négligerons les effets de diffraction ou de canalisation des rayons $\mathrm{X}$ et des photoélectrons.

c) La réflexion et la réfraction des rayons $X$ à l'interface anticathode objet seront décrites en utilisant l'électromagnétisme classique.

d) Les rayons $\mathrm{X}$ et les électrons suivent un affaiblissement exponentiel le long de leur trajet.

Dans l'objet cette longueur d'atténuation sera donnée par $\Lambda_{\mathrm{x}}$ pour les rayons $\mathrm{X}$ et par $\lambda_{\mathrm{e}}$ pour les photoélectrons.

e) Nous négligerons la réflexion des rayons $\mathrm{X}$ et des électrons à l'interface objet vide.

$f$ ) Enfin comme nous cherchons à réduire les dimensions de la source de photoélectrons, nous évaluerons l'intensité électronique comprise entre $\theta_{0}$ et $\theta_{0}+\Delta \theta$ ( $\Delta \theta$ étant l'angle d'acceptance de l'analyseur) à partir de la distribution angulaire de celle-ci plutôt qu'en tenant compte, comme Henke et Fadley, de la projection de l'ouverture d'entrée de l'analyseur A sur la surface de l'échantillon.

Compte tenu de ces approximations et en appelant $\mu$ le coefficient d'auto-absorption linéaire du rayonnement $\mathrm{X}$ dans l'anticathode, l'intensité $\mathrm{d} I X$ traversant une couronne circulaire de cote $Z$ et caractérisée par $\Phi$ et $\mathrm{d} \Phi$ (voir Fig. 4) est égale à : 


$$
\begin{aligned}
\mathrm{d} I_{\mathbf{x}}=\left[\frac{Q}{2} \cos \Phi\right. & \left.\exp -\frac{\mu h}{\sin \Phi} \mathrm{d} \Phi\right] \times \\
\times & {\left[(1-R) \frac{\sin \Phi}{\sin \Phi^{\prime}} \exp -\frac{Z}{\Lambda_{\mathrm{x}} \sin \Phi^{\prime}}\right] }
\end{aligned}
$$

( $R$ représentant le coefficient de réflexion des rayons $\mathrm{X}$ à l'interface anticathode objet).

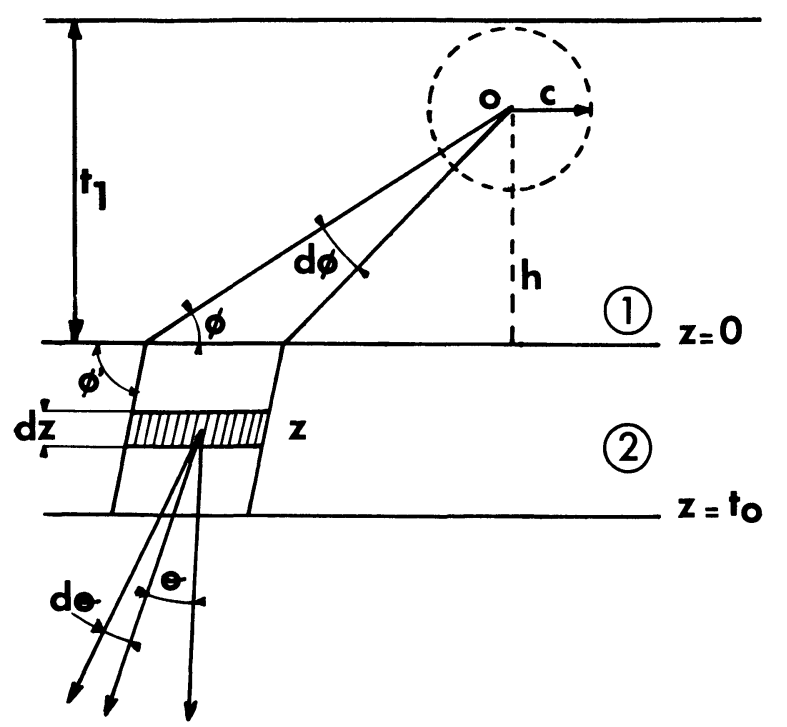

Fic. 4. - Schéma précisant les notations utilisées. 1. Anticathode. 2. Objet.

Le nombre de photoélectrons émis par seconde entre $\theta$ et $\theta+\mathrm{d} \theta$ dans la tranche d'épaisseur $\mathrm{d} z$ (aire hachurée sur la Fig. 4) sera donné par l'expression suivante formellement comparable à l'expression (4) de Fadley et coll.

$$
\begin{aligned}
\mathrm{d} N_{\mathrm{e}}=\left[\mathrm{d} I_{x}\right] \cdot\left[\frac{\rho \mathrm{d} z}{\sin \Phi^{\prime}}\right] \cdot\left[\frac{\mathrm{d} \sigma_{\mathrm{nl}}}{\mathrm{d} \Omega} 2 \pi \sin \theta \mathrm{d} \theta\right] . \\
\cdot\left[\exp -\frac{t_{0}-z}{\lambda_{\mathrm{e}} \cos \theta}\right]
\end{aligned}
$$

dans laquelle $\rho$ représente le nombre par unité de volume des atomes pris en considération, $\mathrm{d} \sigma_{\mathrm{n} 1} / \mathrm{d} \Omega$ la section efficace photoélectrique différentielle pour la sous-couche atomique $\mathrm{nl}$ tandis que le dernier terme traduit l'affaiblissement des photoélectrons se propageant à incidence oblique.

L'intégration de (7) sur l'épaisseur de l'objet conduit au résultat suivant :

$$
\begin{aligned}
\mathrm{d} N_{\mathrm{e}}(\theta, \varphi)=Q \rho \pi & \sin \theta \exp -\frac{\mu h}{\sin \Phi} \cdot \\
& \cdot(1-R) \cdot \frac{\sin \Phi \cos \Phi}{\sin ^{2} \Phi^{\prime}} \cdot \frac{\mathrm{d} \sigma_{\mathrm{n}} \mathrm{d} \theta \mathrm{d} \varphi}{\mathrm{d} \Omega} \\
& \cdot \frac{\Lambda_{\mathrm{x}} \lambda_{\mathrm{e}} \cos \theta \sin \Phi^{\prime}}{\Lambda_{\mathrm{x}} \sin \Phi^{\prime}-\lambda_{\mathrm{e}} \cos \theta} \\
& \cdot\left[\exp -\frac{t_{0}}{\Lambda_{\mathrm{x}} \sin \Phi^{\prime}}-\exp -\frac{t_{0}}{\lambda_{\mathrm{e}} \cos \theta}\right] .
\end{aligned}
$$

Cette expression est assez compliquée et se prête mal à l'intégration suivant $\varphi$, aussi comme nous ne cherchons ici qu'à fixer un ordre de grandeur raisonnable de l'intensité photoélectronique entrant dans l'analyseur, nous allons la simplifier en faisant les approximations supplémentaires suivantes :

g) Nous négligerons la réflexion et la réfraction des rayons $\mathrm{X}$ à l'interface anticathode objet $\left(\Phi=\Phi^{\prime}\right)$ : cela conduit à ne pas prendre en compte des effets qui ont été étudiés en détail par Henke et qui sont physiquement intéressants mais qui, se manifestant à incidence presque rasante, sont d'un poids relativement faible dans la mesure où les photons $\mathrm{X}$ correspondants sont fortement atténués par leur long trajet dans l'anticathode.

Pour cette raison et compte tenu des ordres de grandeur respectifs de $\Lambda_{\mathrm{x}}$ et $\lambda_{\mathrm{e}}$, nous négligerons, dans (8), $\lambda_{\mathrm{e}} \cos \theta$ devant $\Lambda_{\mathrm{x}} \sin \varphi$.

h) La section efficace de photoionisation est de la forme :

$$
\frac{\mathrm{d} \sigma_{\mathrm{n} 1}}{\mathrm{~d} \Omega}=\frac{\sigma_{\mathrm{nl}}}{4 \pi}\left[1+\frac{\beta}{2}\left(\frac{3}{2} \sin ^{2} \alpha-1\right)\right]
$$

dans laquelle $« \alpha$ » est l'angle que fait la direction des photons $X$ incidents (non polarisés) avec la direction que prend le photoélectron éjecté et $\beta$ est un paramètre d'asymétrie qui dépend du moment angulaire de l'électron sur son orbite.

Par souci de simplification, nous ne tiendrons pas compte de la polarisation éventuelle des rayons $\mathrm{X}$ et nous supposerons que l'effet photoélectrique est isotrope dans l'échantillon.

i) Bien que ce calcul, pour une épaisseur d'objet quelconque, ne présente pas de difficulté, nous donnerons, pour simplifier, l'intensité photoélectronique dans l'hypothèse où l'épaisseur de l'objet est optimisée : comme la partie utile de l'objet est située à l'interface objet vide sur une profondeur de l'ordre de $\lambda_{\mathrm{e}}$, il est souhaitable de choisir cette épaisseur de telle manière que, tout en étant supérieure à $\lambda_{\mathrm{e}}$, elle soit inférieure à la longueur d'atténuation des rayons $X$ dans l'objet $\left(\Lambda_{x}\right.$ de 1000 à $10000 \AA$ ) afin que ceux-ci ne soient pas trop fortement atténués avant d'atteindre cette zone utile ; « $t_{0} »$ devra donc satisfaire à l'inégalité

$$
\Lambda_{\mathrm{x}} \gg t_{0}>\lambda_{\mathrm{e}}
$$

et aura un ordre de grandeur comparable à l'épaisseur des échantillons en microscopie électronique $\left(t_{0} \simeq 100 \AA\right)$.

Dans ces conditions, le nombre de photoélectrons quasiment monocinétiques $(\Delta E=1 \mathrm{eV})$ et qui pénètrent dans l'analyseur $(\theta, \theta+\Delta \theta)$ par seconde est donné par :

$$
\begin{array}{r}
N(\theta, \Delta \theta)=\left[\frac{Q}{2}\right]\left[\rho \cdot \frac{\sigma_{\mathrm{n} 1}}{2} \cdot \lambda_{\mathrm{e}} \cos \theta\right][\sin \theta \Delta \theta] \times \\
\times\left[\int_{\pi / 2}^{0} \frac{\cos \Phi}{\sin \Phi} \exp -\frac{\mu h}{\sin \Phi} \mathrm{d} \Phi\right] .
\end{array}
$$


Dans cette expression, les différents termes entre crochets ont la signification suivante :

- le premier terme représente l'intensité $X$ créée par l'anticathode et se dirigeant vers l'objet,

- le deuxième terme traduit le rendement quantique de l'effet photoélectrique pour l'excitation de la souscouche nl d'un élément donné, les électrons élastiques correspondants étant issus d'une épaisseur utile égale à $\lambda_{\mathrm{e}} \cos \theta$.

Si l'objet est constitué d'un seul élément et si une seule soùs-couche est susceptible d'être excitée, ce terme

$$
\rho \frac{\sigma}{2} \lambda_{\mathrm{e}} \cos \theta
$$

est sensiblement égal à :

$$
\frac{\lambda_{\mathrm{e}}}{2 \Lambda_{\mathrm{x}}} \cos \theta
$$

(résultat comparable à celui obtenu par une évaluation antérieure [4]),

- le troisième terme inclut la transparence de l'analyseur,

- la quantité sous l'intégrale dans le dernier terme représente la répartition angulaire de l'intensité $X$ éclairant l'objet : son évolution en fonction de $\Phi$ sera discutée dans le chapitre résolution spatiale sur laquelle elle agit directement.

L'intégrale définie tient compte de l'auto-absorption $\mathrm{X}$ dans l'anticathode et de l'influence de la direction de propagation des photons dans l'objet sur l'intensité photoélectronique $(\cos \Phi)$.

L'évolution en fonction de $\mu h$ du résultat numérique correspondant est représentée sur la figure 5.

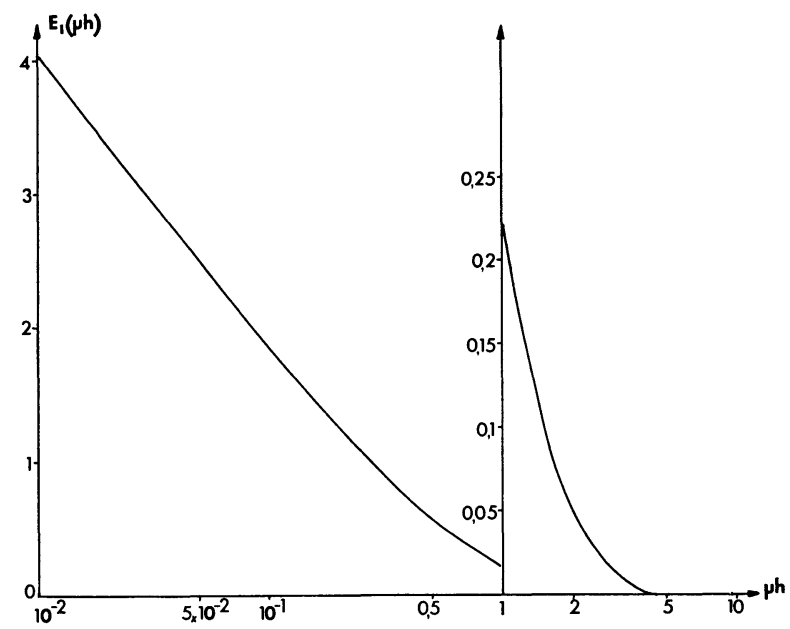

FIG. 5. - Influence de l'auto-absorption X dans l'anticathode.

3.4 OPTIMISATION DES PARAMÈTRES. - 3.4.1 Nature del'anticathode. - La radiation $\mathrm{X}$ excitatrice est caractérisée par l'énergie $E$ et la largeur énergétique à mihauteur $(\Delta E)$ des photons émis.

En spectroscopie des photoélectrons, on emploie habituellement les radiations caractéristiques $\mathrm{AlK}_{\alpha}$ et $\mathrm{MgK}_{\alpha}$ mais Krause [15] a signalé récemment l'existence de radiations de plus grande longueur d'onde permettant d'obtenir une résolution énergétique satisfaisante $(\Delta E \leqslant 1,5 \mathrm{eV})$ ce sont les radiations suivantes :

$$
\begin{gathered}
\mathrm{YM}_{\xi}(E=132,3 \mathrm{eV}, \Delta E \simeq 0,47 \mathrm{eV}) \\
\mathrm{ZnM}_{\xi}(E=151,4 \mathrm{eV}, \Delta E=0,77 \mathrm{eV}) \\
\operatorname{NbM}_{\xi}(E=171,4 \mathrm{eV}-\Delta E=1,21 \mathrm{eV})
\end{gathered}
$$

et

$$
\operatorname{MoM}_{\xi}(E=192,3 \mathrm{eV} \text { et } \Delta E \simeq 1,53 \mathrm{eV}) .
$$

Bien que les lois régissant la production de telles radiations (lois analogues à l'expression (15) établie pour certaines radiations du type $\mathrm{K}_{\alpha}$ ou $\mathrm{L}_{\alpha}$ ) ne soient pas connues, nous tenterons d'estimer, à partir des observations de Krause, l'intensité photoélectronique que l'on peut espérer atteindre en les utilisant, car leur emploi peut s'avérer précieux pour améliorer la résolution spatiale du dispositif préconisé.

3.4.2 Optimisation de l'émission photoélectronique. - La radiation caractéristique étant choisie, les seules grandeurs extérieures sur lesquelles on peut agir, dans l'expression (11), pour optimiser l'intensité photoélectronique sont $Q$ et $h$.

Nous avons vu au paragraphe 3.2 (5) que la distance « $h$ » optimale entre la source $\mathrm{X}$ et l'objet correspondait à une épaisseur $t_{1}$ de l'anticathode donnée par

$$
t_{1}=h+z_{\mathrm{D}}=2 z_{\mathrm{m}}-z_{\mathrm{D}}
$$

soit

$$
h=2\left(z_{\mathrm{M}}-z_{\mathrm{D}}\right) \text {. }
$$

Si l'on tient compte : d'une part, de l'expression (14) déduite de la loi de décélération des électrons (Castaing [8] (24)).

$$
z_{\mathrm{M}}=0,033\left(V_{0}^{1,7}-V_{\mathbf{k}}^{1,7}\right) \frac{A}{\rho Z}
$$

$\left(z_{\mathrm{m}}\right.$ en $\mu, V_{0}$ et $V_{\mathrm{k}}$ en $\mathrm{kV}, \rho$ densité volumique de la cathode en $\mathrm{g} / \mathrm{cm}^{3}$ ) et, d'autre part, de la relation empirique établie par Coslett [16]

$$
\frac{z_{\mathrm{D}}}{z_{\mathrm{M}}}=\frac{12}{Z+8}
$$

on constate que cette valeur optimale est fonction de la tension d'accélération $V_{0}$ des électrons incidents.

Pour calculer le nombre total $(Q)$ de photons X émis par seconde, on peut utiliser la relation (14) :

$$
Q=\frac{I_{0}}{q} N
$$

$\left(q=\right.$ charge de l'électron : $1,6 \times 10^{-19}$ C) dans laquelle $N$ est le nombre de photons X émis par électron incident et qui peut être calculé à partir de la formule de Green et Coslett [17] :

$$
\frac{N}{4 \pi}=\frac{\mathcal{N}}{4 \pi}\left(\frac{V_{0}}{V_{\mathrm{k}}}-1\right)^{1 ; 63}
$$


(avec $\mathcal{N}$ un coefficient ne dépendant que de la nature de l'anticathode : par exemple $\mathcal{N} / 4 \pi=1,4 \times 10^{-5}$ pour $\mathrm{AlK}_{\alpha}$ ).

On constate donc que $Q$ dépend simultanément de $V_{0}$ et $I_{0}$ mais, en fait, ces 2 variables ne sont pas complètement indépendantes si on cherche à les maximaliser ; il faut, en effet, tenir compte de ce que la valeur maximale de $I_{0}$ sera conditionnée par l'une des deux limites physiques exprimées par les formules (16) et (17) soit :

- D'une part, l'intensité $I_{0}$ électroniquement admissible dans une sonde de diamètre $d_{0}$ compte tenu des aberrations du système d'optique électronique délivrant le faisceau (Castaing [8]) à savoir :

$$
\begin{aligned}
& I_{0}=0,016 \quad V_{0} \quad d_{0}^{8 / 3} \\
& (\mu \mathrm{A}) \quad(\mathrm{kV})(\mu \mathrm{m})
\end{aligned}
$$

dans laquelle le coefficient numérique $(0,016)$ relatif à un filament de tungstène porté à $2700 \mathrm{~K}$ pourrait être amélioré par l'emploi de cathodes en $\mathrm{LaB}_{6}$ (Loeffler [18]) ou de canons à émission de champ (pour des sondes de très petits diamètres).

- Et d'autre part, l'intensité qui peut thermiquement supporter l'anticathode sans être détériorée, c'est-à-dire (Castaing)

$$
V_{0} I_{0}=\frac{2 \mathrm{~J} \pi K t_{1} \Delta T}{0,5+\log \frac{d_{1}}{d_{0}}}
$$

( $\Delta T=T_{0}-T_{1}$, élévation de température entre le diamètre $d_{1}$ maintenu à la température $T_{1}$ et le point le plus chaud $T_{0}$ de l'anticathode ayant la forme d'une lame à faces parallèles, $K$ la conductibilité thermique de l'anticathode). Puisque toutes les variables ajustables s'expriment en fonction de $V_{0}$, nous allons calculer numériquement, au paragraphe suivant, l'intensité photoélectronique que l'on peut atteindre pour différentes valeurs de $V_{0}$ et nous pourrons en déduire les meilleures conditions de fonctionnement.

3.5 ESTIMATIONS NUMÉRIQUES. - 3.5.1 Ordre de grandeur de l'intensité photoélectronique admissible avec les radiations $\mathrm{AlK}_{\alpha}$. - Afin de préciser les intensités photoélectroniques que l'on peut espérer atteindre en utilisant une anticathode d'aluminium, nous allons évaluer ces intensités à partir de l'expression (11) et pour différentes valeurs de la tension d'accélération des électrons incidents [compte tenu des relations (12) à (15)].

Comme les sections efficaces partielles de photoionisation n'ont fait l'objet que d'études très restreintes (Wuillemier et Krause [19], Kemeny et coll. [20]) nous évaluerons l'intensité photoélectronique relative à une raie de l'or en supposant que sa section efficace de photoionisation est environ le dizième de la section efficace totale (laquelle contribue presque exclusivement à ces longueurs d'onde, à l'atténuation des rayons $X$ dans la matière) soit $\sigma_{\mathrm{n} 1} \simeq 16 \times 10^{-20} \mathrm{~cm}^{2}$ et $\rho=6 \times 10^{22}$ at $/ \mathrm{cm}^{3}$.

Avec un analyseur miroir cylindrique analogue à celui mis au point par Citrin et coll. $\left(\theta_{0}=60^{\circ}\right)$ et compte tenu de la longueur d'atténuation $\left(\lambda_{\mathrm{e}} \simeq 25 \AA\right)$ des photoélectrons Au $4 \mathrm{f}_{7 / 2}$ excités par $\mathrm{AlK}_{\alpha}$ (Fadley et coll. [14]), le rendement quantique de l'effet photoélectrique pour cette raie $-2^{\mathrm{e}}$ terme de $(11)-$ sera de l'ordre de $0,6 \times 10^{-3}$.

La résolution énergétique sera meilleure que $1 \mathrm{eV}$ en prenant pour $\Delta \theta$ ( $3^{\mathrm{e}}$ terme) une valeur sensiblement égale à $2 \times 10^{-2}$ (Citrin et coll.).

L'épaisseur de l'objet sera supposée être de l'ordre de $100 \AA$ de telle manière que l'on puisse la négliger (10) dans l'estimation du $4 \mathrm{e}$ terme tout en conservant la précaution $h=2 c(5)$.

La valeur de l'exponentielle intégrale correspondante $\left[E_{1}(\mu h)\right]$ sera extraite de la littérature (Handbook of chemistry and physics) et dans le premier terme $(\varphi / 2)$ la valeur de l'intensité $I_{0}$ incidente sur l'anticathode sera la valeur la plus faible obtenue à partir des relations (16) et (17).

Dans l'expression (17), on ne tolérera au centre de la sonde qu'une élévation maximale de température égale à la moitié de la température de fusion de l'anticathode (soit $\Delta T=300^{\circ} \mathrm{C}$ ) tout en admettant que la température ambiante règne à la périphérie de l'anticathode sur une circonférence de diamètre $d_{1} \simeq 20 \mathrm{~mm}$.

Les résultats numériques ainsi obtenus sont consignés dans le tableau I.

On observera, qu'aux basses tensions, l'intensité photoélectronique, conditionnée essentiellement par la limite électronique, croît très rapidement avec la tension $V_{0}$.

Au-delà de $14 \mathrm{keV}$, l'intensité photoélectronique est conditionnée par un compromis entre la limite thermique d'une part et l'auto-absorption du rayonnement dans l'anticathode d'autre part, et elle passe par un maximum qui correspond à l'optimisation, déjà mentionnée antérieurement [4], de la dimension « $h$ » $(h \simeq 1 / \mu)$.

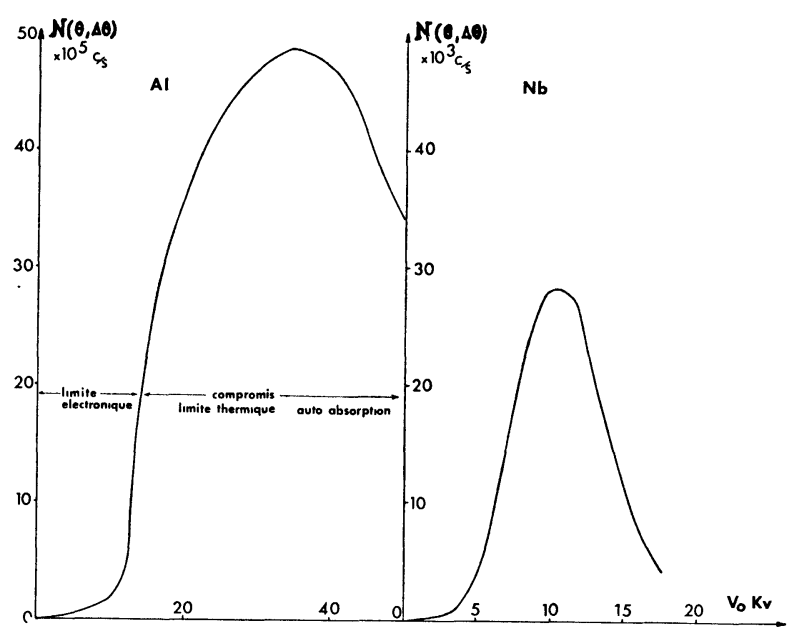

FIG. 6. - Evolution de l'intensité photoélectronique détectée $N(\theta, \Delta \theta)$ en fonction de la tension des électrons incidents. Courbes tracées d'après les tableaux I et III ; a) radiation $\mathrm{AlK}_{\alpha}$ (à gauche) ; b) radiation $\mathrm{NbM}_{\zeta}$ (à droite). 
TABleaux I, II et III

Estimation en fonction de $V_{0}$ (4e colonne) de l'évolution du nombre de photoélectrons/s $N(\theta, \Delta \theta)$ (soit $I_{\mathrm{pe}}$ : dernière colonne) pouvant être détectés et correspondant à l'excitation d'une raie photoélectronique caractérisée par : $\sigma_{\mathrm{n} 1} \approx 16 \times 10^{-20} \mathrm{~cm}^{2}$ (Tableaux I et II), $\sigma_{\mathrm{n} 1} \approx 80 \times 10^{-20} \mathrm{~cm}^{2}$ (Tableau III) avec $\rho=6 \times 10^{22} \mathrm{at} / \mathrm{cm}^{3}$.

Ces tableaux supposent optimisés les autres paramètres ajustables et ils précisent leur valeur :

$t_{1}$ : épaisseur de l'anticathode : $6^{\mathrm{e}}$ colonne.

$d_{0}:$ diamètre de la sonde électronique incidente $: 7 \mathrm{e}$ colonne.

$I_{0}:$ intensité de la sonde incidente en $\mu \mathrm{A}$ puis en élec./s : $8^{\mathrm{e}}$ et $9^{\mathrm{e}}$ colonnes.

$\Delta T$ : échauffement correspondant de l'anticathode : $10^{\mathrm{e}}$ colonne.

$$
\begin{aligned}
& N(\theta, \Delta \theta)=\left[\frac{Q}{2}\right]\left[\frac{\rho \sigma_{\mathrm{T}}}{2} \lambda_{\mathrm{e}} \cos \theta\right][\sin \theta \Delta \theta]\left[\int_{\pi / 2}^{0} \frac{\cos \Phi}{\sin \Phi} \exp -\frac{\mu h}{\sin \Phi} \mathrm{d} \Phi\right] \\
& \begin{array}{llll}
\downarrow & \downarrow & \downarrow & \downarrow \\
I_{\mathrm{pe}} & \stackrel{A}{*} & B & E_{1}(\mu h)
\end{array} \\
& \left(\eta_{\text {p.e. }}\right)
\end{aligned}
$$

\begin{tabular}{|c|c|c|c|c|c|c|c|c|c|c|c|c|c|}
\hline$\mu h$ & $\begin{array}{c}E_{1} \\
(\mu h)\end{array}$ & $\underset{(\mu \mathrm{m})}{h}$ & $\begin{array}{r}V_{0} \\
(\mathrm{kV})\end{array}$ & $\begin{array}{c}N \\
\left(10^{-3}\right)\end{array}$ & $\begin{array}{r}t_{1} \\
(\mu \mathrm{m})\end{array}$ & $\begin{array}{c}d_{0} \\
(\mu \mathrm{m})\end{array}$ & $\begin{array}{c}I_{0} \\
(\mu \mathrm{A})\end{array}$ & $\begin{array}{c}I_{0} \\
\left(10^{13} \mathrm{e} / \mathrm{s}\right)\end{array}$ & $\begin{array}{l}\Delta T \\
\mathrm{OK}\end{array}$ & $\begin{array}{c}Q / 2 \\
\left(10^{10}\right)\end{array}$ & $A$ & $B$ & $\begin{array}{c}I_{\mathrm{pe}} \\
\left(10^{5} \mathrm{e} / \mathrm{s}\right.\end{array}$ \\
\hline & $\overline{0,05}$ & $\overline{19}$ & $\overline{53}$ & 56 & $\overline{32}$ & $\overline{40}$ & $\overline{38}$ & 24 & $\overline{300}$ & $67 \overline{0}$ & $6 \times 10^{-4}$ & $1.7 \times 10^{-2}$ & 34 \\
\hline 1 & 0,22 & 9,5 & 35 & 28 & 16 & 20 & 26 & 16 & 300 & 220 & & $0^{-5}$ & 49 \\
\hline ,5 & 0,56 & 4,7 & 24 & 14,5 & 8 & 10 & 17 & 10,5 & 300 & 76 & & - & 42 \\
\hline 0,2 & 1,22 & 1,9 & 14 & 5,6 & 3,2 & 4 & 9 & 5,6 & 250 & 15 & & - & 19 \\
\hline 0,1 & 1,82 & 0,9 & 9,5 & 2,7 & 1,6 & 2 & 0,9 & 0,6 & 40 & 0,8 & & - & 1,5 \\
\hline 0,05 & 2,45 & 0,5 & 6,5 & 1,25 & 0,8 & 1 & 0,1 & 0,06 & 6 & 0,04 & & - & 0,08 \\
\hline
\end{tabular}

\section{TABLEAU I}

Radiation $\mathrm{X}$ utilisé : $\mathrm{AlK}_{\alpha}$

\begin{tabular}{|c|c|c|c|c|c|c|c|c|c|c|c|c|}
\hline$\mu h$ & $\begin{array}{c}E_{1} \\
(\mu h)\end{array}$ & $\begin{array}{c}h \\
(\mu \mathrm{m})\end{array}$ & $\begin{array}{r}V_{0} \\
(\mathrm{kV})\end{array}$ & $\begin{array}{c}N \\
\left(10^{-3}\right)\end{array}$ & $\begin{array}{c}t_{1} \\
(\mu \mathrm{m})\end{array}$ & $\begin{array}{c}d_{0} \\
(\mu \mathrm{m})\end{array}$ & $\begin{array}{c}I_{0} \\
(\mu \mathrm{A})\end{array}$ & $\begin{array}{c}I_{0} \\
\left(10^{13} \mathrm{e} / \mathrm{s}\right)\end{array}$ & $\begin{array}{l}\Delta T \\
\mathrm{OK}\end{array}$ & $\begin{array}{c}Q / 2 \\
\left(10^{10}\right)\end{array}$ & $A B$ & $\begin{array}{c}I_{\mathrm{pe}} \\
\left(10^{5} \mathrm{e} / \mathrm{s}\right)\end{array}$ \\
\hline $1 \overline{5}$ & $\overline{0,1}$ & $\overline{22,5}$ & $\overline{49}$ & $\overline{56}$ & $\overline{39}$ & 45 & 22 & $\overline{14}$ & $\overline{300}$ & $40 \overline{0}$ & $\overline{10^{-5}}$ & 40 \\
\hline & 0,22 & 15 & 38 & 37 & 26 & 30 & 18 & 11 & 300 & 200 & - & 44 \\
\hline 0,5 & 0,56 & 7,5 & 26 & 19 & 13 & 15 & 12 & 7,5 & 300 & 70 & - & 39 \\
\hline 0,2 & 1,22 & 3 & 15 & 75 & 5 & 6 & 7 & 4,3 & 300 & 16 & - & 19 \\
\hline 0,1 & 1,82 & 15 & 10 & 3,5 & 2,6 & 3 & 3 & 1,9 & 180 & 33 & - & 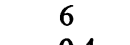 \\
\hline 0,05 & 2,45 & 0,75 & 7 & 1,8 & 1,3 & 1,5 & 0,33 & 0,2 & 30 & 0,18 & - & 0,4 \\
\hline
\end{tabular}

\section{TABLEAU II}

Radiation X utilisé $\mathrm{MgK}_{\alpha}$

\section{TABLEAU III}

Radiation X utilisé $\mathrm{NbM}_{\xi}$

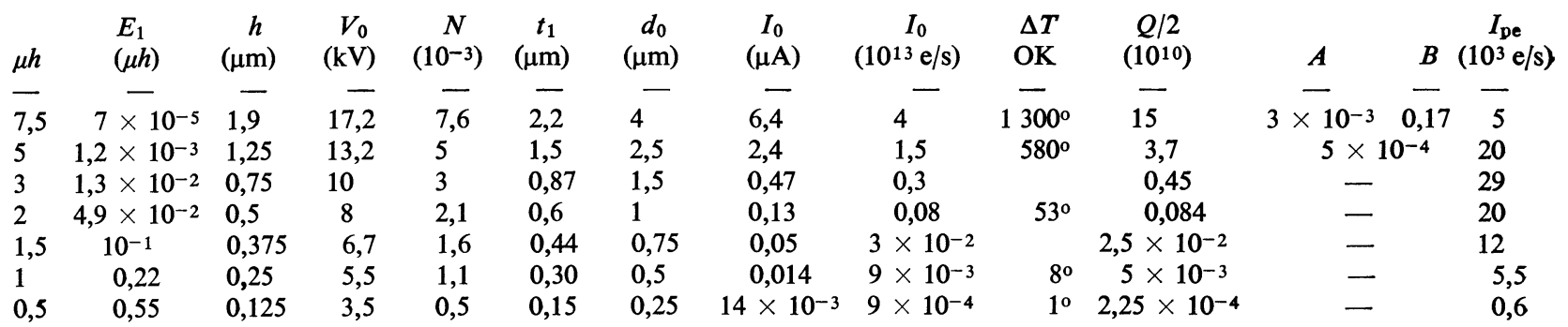


Comme ce maximum est assez peu marqué (Fig. 6), le choix de la tension de fonctionnement n'obéit pas à des impératifs très critiques et un taux de comptage de l'ordre de $10^{6} \mathrm{cps} / \mathrm{s}$ pourra, théoriquement, être obtenu dans un intervalle de tension compris entre 12 et $40 \mathrm{keV}$ [si toutefois tous les autres paramètres sont ajustés à leur valeur optimisée en conformité avec la tension choisie et en supposant que la relation (15) puisse être extrapolée jusqu'à des tensions aussi élevées].

Remarques. - 1) Le tableau I permet de fixer des ordres de grandeur mais il ne prétend pas donner des intensités photoélectroniques exactes car les sections efficaces partielles de photoionisation varient en fonction de la sous-couche et de l'élément considéré, de plus la non-planéité de la surface de l'échantillon peut provoquer, en augmentant l'aire émissive, un accroissement de l'intensité photoélectronique (Fadley et coll. [14]).

2) L'expression (4) reliant le diamètre de la sonde électronique incidente à la tension d'accélération des électrons (par l'intermédiaire des relations (12) et (13)) n'a été établie que pour justifier le modèle de la source $X$ ponctuelle et apprécier ainsi de façon simple l'autoabsorption du rayonnement $\mathrm{X}$ dans l'anticathode ; en ce qui concerne les intensités photoélectroniques émises entre $\theta_{0}$ et $\theta_{0}+\Delta \theta$, on peut donc s'affranchir éventuellement de cette condition en choisissant un diamètre de sonde (et donc de source $\mathrm{X}$ ) plus important afin d'accroître $I_{0}$ et donc $N(\theta, \theta+\Delta \theta)$ (dans le cadre des limites physiques imposées par les relations (16) et (17)) ; toutefois, dans ce cas, l'évaluation du terme correspondant à $E_{1}(\mu h)$ est assez malaisée.

3) Bien qu'ayant choisi une source $X$ quasiment ponctuelle, nous verrons au chapitre de la résolution spatiale que la source photoélectronique présente une certaine extension latérale et donc ne saurait être, elle, considérée comme ponctuelle : un certain nombre de photoélectrons bien qu'émis dans l'angle compris entre $\theta_{0}$ et $\theta_{0}+\Delta \theta$ d'acceptance de l'analyseur, ne peuvent pénétrer dans celui-ci parce qu'ils sont émis d'un point de l'objet trop éloigné de l'axe de révolution du système. Autrement dit, comme la résolution $R_{0}$ de tout spectromètre (quel que soit le modèle d'analyseur utilisé) est déterminée par le terme d'aberration sphérique $R(\Delta \theta)$ d'une part, et le terme d'aberration $R(\Delta S)$ lié aux dimensions finies de l'objet source d'autre part, une partie de l'intensité photoélectronique évaluée ci-dessus ne pourra être détectée.

A fortiori cette perte d'intensité sera accrue par l'utilisation d'une source $X$ elle-même étalée : c'est ainsi que dans le montage de Citrin et coll. [6], l'utilisation d'un canon à électrons ayant un filament enroulé en spirale ( $2 \mathrm{~cm}$ de diamètre) entraîne, malgré une optique probablement réductive, une extension de la source $X$ et donc de la source photoélectronique très largement supérieure au rayon du diaphragme d'entrée de l'analy- seur $(1 \mathrm{~mm})$ (diaphragme destiné à conférer à celui-ci une résolution énergétique inférieure à $1 \mathrm{eV}$ ).

C'est à cause de cette dernière remarque que nous n'avons pas malheureusement pu comparer les résultats expérimentaux de Citrin et coll. avec ceux que l'on peut déduire de l'expression (11) en tenant compte de leurs conditions expérimentales.

3.5.2 Evaluation des intensités photoélectroniques admissibles en utilisant des anticathodes de magnésium et niobium. - a) Magnésium. - Nous avons appliqué au rayonnement $\mathrm{MgK}_{\alpha}$ la démarche exposée ci-dessus pour $\mathrm{AlK}_{\alpha}$ en adoptant pour $\mathcal{N}$ (15) la valeur déduite de la figure 6 de l'article de Green et Coslett [17] soit $\mathcal{N}=1,2 \times 10^{-5}$ photon/stérad. électron et pour la conductivité thermique $K(17)$ la valeur extraite de la littérature (Smithells [22] $K_{\mathrm{Mg}}=0,13 \mathrm{cgs}$ ), ceci nous a permis d'établir le tableau II dans lequel on observera que les intensités photoélectroniques théoriquement admissibles sont en tout point comparables à celles déjà évaluées à partir de $\mathrm{AlK}_{\alpha}$.

b) Niobium. - Pour évaluer les intensités que l'on peut espérer atteindre en utilisant une anticathode en niobium, nous avons dû, faute de données plus précises, faire les approximations suivantes :

- Nous avons admis que la relation (15) établie pour certaines radiations du type $K_{\alpha}, L_{\alpha}$ et $M_{\alpha}$ était aussi valable pour les radiations du type $\mathbf{M}_{\xi}$.

- A partir des observations de Krause [15] montrant qu'à $6 \mathrm{keV}$, l'intensité $I(X)$ émise par une anticathode en niobium avec un angle d'émergence $\langle\alpha$ » de $7^{\circ}$ puis de $25^{\circ}$ était successivement le quart puis la moitié de l'intensité de la radiation $\mathrm{MgK}_{\alpha}$ (produite dans les mêmes conditions), nous avons pu établir qu'à $6 \mathrm{kV}$ l'intensité avec les 2 types d'anticathode était sensiblement la même, compte tenu de l'auto-absorption des rayonnements : nous avons calculé, à partir des relations (12) et (13), la profondeur à laquelle se produisait l'émission $X$ dans chacune des anticathodes puis, à partir d'une relation de type

$$
I_{\alpha}=I_{0} \exp -\frac{\mu z_{0}}{\sin \alpha},
$$

nous avons obtenu que

$$
I_{0}\left(\mathrm{MgK}_{\alpha}\right) \simeq I_{0}\left(\mathrm{NbM}_{\xi}\right)
$$

avec $\mu_{\mathrm{Mg}} \simeq 1 / 15 \mu \mathrm{m}$ et $\mu_{\mathrm{Nb}} \simeq 1 / 0,25 \mu \mathrm{m}$ (Veigele [21]).

Nous avons ainsi estimé que le coefficient $\mathcal{N}$ dans la relation (15) était de l'ordre de $3,4 \times 10^{-7}$ photon/stérad. électron.

A partir des tables de sections efficaces globales (Veigele [21]), nous avons estimé que la section efficace de photoionisation de la sous-couche $4 \mathrm{f}^{7 / 2}$ de l'or était 5 fois plus élevée pour des photons de $170 \mathrm{eV}$ d'énergie que pour des photons ayant une énergie supérieure à $1000 \mathrm{eV}$ soit $\sigma_{\mathrm{n} 1} \simeq 80 \times 10^{-20} \mathrm{~cm}^{2}$ (estimation réellement minimale si l'on se réfère par exemple aux gains allant de 50 à $10^{3}$ observés pour les sous-couches $2 \mathrm{~s}$ et $2 p$ du Néon par Wuillemier et Krause [19]), par 
contre nous n'avons pas tenu compte du fait que la profondeur d'extraction dépend de l'énergie cinétique et nous avons conservé $\lambda_{\mathrm{e}}=25 \AA$.

L'énergie cinétique maximale des électrons diminuant d'un coefficient sensiblement égal à 10 dans la substitution des anticathodes, nous avons admis que l'angle d'acceptance des électrons dans l'analyseur $\Delta \theta$ pouvait être 10 fois supérieur (la résolution énergétique restant meilleure que l'électron-volt).

Compte tenu de ces estimations, nous avons pu dresser le tableau III dans lequel on peut observer que, l'influence de l'auto-absorption des X devenant notable, il faut choisir des anticathodes très minces, ce qui entraîne l'emploi de sondes électroniques ayant des diamètres plus petits que celles utilisées avec $\mathrm{AlK}_{\alpha}$ et, au total, un nombre de photoélectrons $N(\theta, \Delta \theta)$ environ 100 fois plus faible que celui obtenu avec des radiations plus classiques.

4. Résolution spatiale et analyse quantitative. 4.1 RÉSOlUtion SPATIALE. - La source X ponctuelle éclaire tout l'objet et, comme nous l'avons mentionné plus haut, tout point du plan objet supposé homogène peut émettre des photoélectrons.

Néanmoins, il est qualitativement évident que cette émission n'est pas uniforme sur toute la surface de l'échantillon mais qu'au contraire la majeure partie des photoélectrons émis provient d'une petite aire circulaire centrée sur l'objet en un point $O^{\prime}$ situé sous la source ponctuelle $O$ émettant les rayons X $\left(O O^{\prime}: h\right)$. Il est aisé de prévoir, en particulier, que les dimensions de cette aire émissive seront d'autant plus réduites que la distance entre la source $X$ et l'objet sera faible ; en contrepartie, si l'on réduit cette distance $(h)$, on sera obligé d'opérer avec des tensions d'accélération des électrons incidents plus faibles et, au total, une amélioration de la résolution spatiale se traduira logiquement par une diminution de l'intensité des signaux détectés.

Quantitativement, le nombre de photoélectrons émis par une aire circulaire centrée en $O^{\prime}$ et de rayon $R$ sera donné par une expression identique à l'expression (11) mais pour laquelle l'intégration sur l'angle $\Phi\left[E_{1}(\mu h)\right.$ quatrième terme $]$ ne s'effectuera plus entre $\Phi_{1}=\pi / 2$ et $\Phi_{2}=0$ mais entre $\Phi_{1}=\pi / 2$ et $\Phi_{2}=\operatorname{Arctg} h / R$.

L'évolution de cette dernière intégrale, soit

$$
\int_{\pi / 2}^{\operatorname{Arctg} h / R} \frac{\cos \Phi}{\sin \Phi} \exp -\frac{\mu h}{\sin \Phi} \mathrm{d} \Phi
$$

en fonction du rapport $R / h$ et pour différentes valeurs de la quantité $\mu h($ de $\mu h=0,05$ à $\mu h=2)$ est indiquée sur les courbes suivantes : figures $7 a, b, c, d, e$, sur lesquelles nous avons symbolisé par des flèches les rapports $R / h$ qui correspondent à des fractions croissantes (de $50 \%$ à $90 \%$ ) de l'intensité photoélectronique totale.

A partir de ces résultats et en considérant chaque type d'anticathode, il est désormais possible de donner l'évolution du rayon des aires circulaires émettant respectivement $50 \%, 70 \%$ et $80 \%$ de l'intensité photoélectronique détectée (soit $\boldsymbol{R}_{50 \%}, \boldsymbol{R}_{70 \%}, \boldsymbol{R}_{80 \%}$ ) en fonction de la distance $" h$ » entre la source et l'objet : pour cela, il suffit, sur les courbes des figures $7 a$, $b, c, d, e$, de repérer les abscisses $(R / h)$ correspondant à l'émission d'une fraction donnée de l'intensité photoélectronique et de tenir compte de la valeur numérique du coefficient d'auto-absorption linéaire du rayonnement $X$ dans l'anticathode (soit $\mu_{\mathrm{A} 1}=1 / 8,5 \mu \mathrm{m}^{-1}$ pour $\mathrm{AlK}_{\alpha}$ et $\mu_{\mathrm{Nb}}=1 / 0,25 \mu \mathrm{m}^{-1}$ pour $\left.\mathrm{NbM}_{\xi}\right)$.
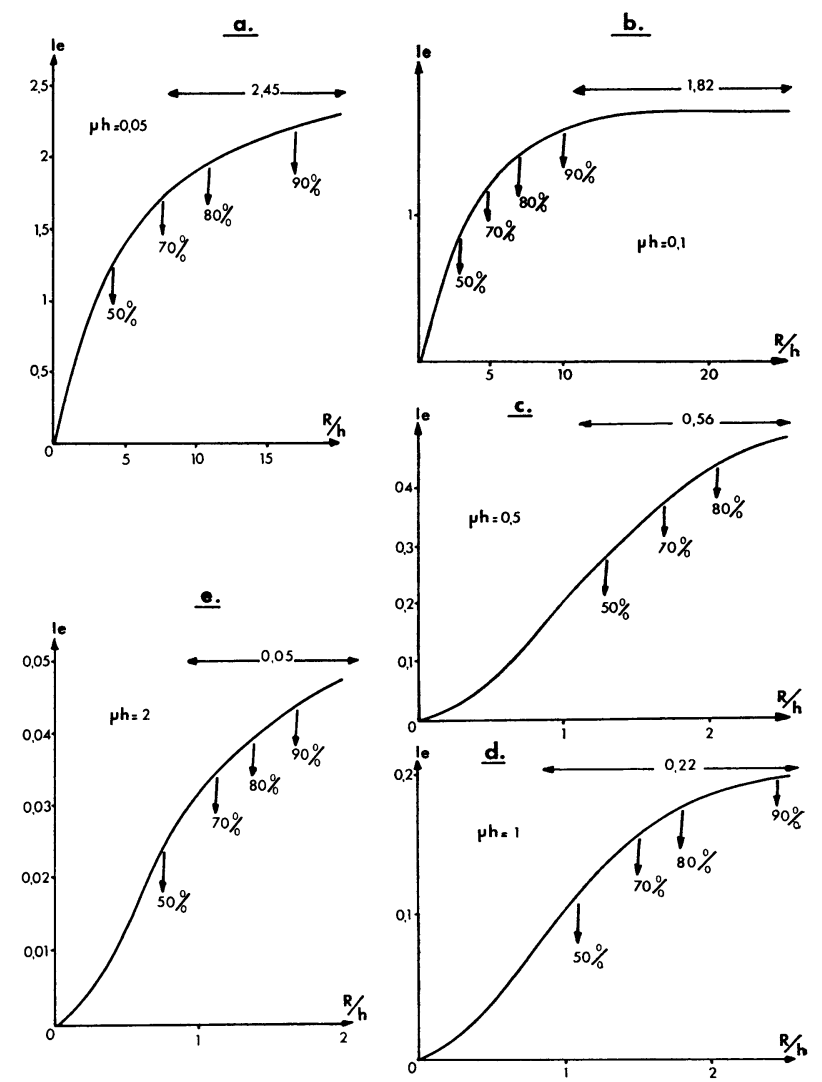

FIG. 7. - Evolution relative de l'intensité photoélectronique détectée en fonction de $R / h$ ( $R$ étant le rayon croissant de l'aire circulaire qui émet les photoélectrons). Cela correspond aussi à l'évolution de

$$
\int_{0}^{\operatorname{Arctg} R / h} \frac{\cos \Phi}{\sin \Phi} \exp -\frac{\mu h}{\sin \Phi} \mathrm{d} \Phi
$$

en fonction de $R / h$ et pour différentes valeurs de $\mu h$ $(a: \mu h=0,05 ; b: \mu h \simeq 0,1 ; c: \mu h \simeq 0,5 ; d: \mu h \simeq 1$; $e: \mu h \simeq 2$ )

Les résultats obtenus sont représentés sur les figures $8 a$ pour l'aluminium et $8 b$ pour le niobium avec une double échelle des abscisses qui permet d'établir la correspondance entre le paramètre $h$ et la tension d'accélération optimale pour les électrons incidents (comme en pratique, c'est le choix de l'épaisseur $t_{1}$ de l'anticathode qui, pour une tension $V_{0}$ donnée, entraîne, par la relation (5), la valeur du paramètre $h$, nous avons aussi représenté sur ces figures la correspondance optimale entre $h$ et $t_{1}: t_{1}=1,66 h$ pour l'aluminium et $t_{1}=1,16 h$ pour le niobium). 


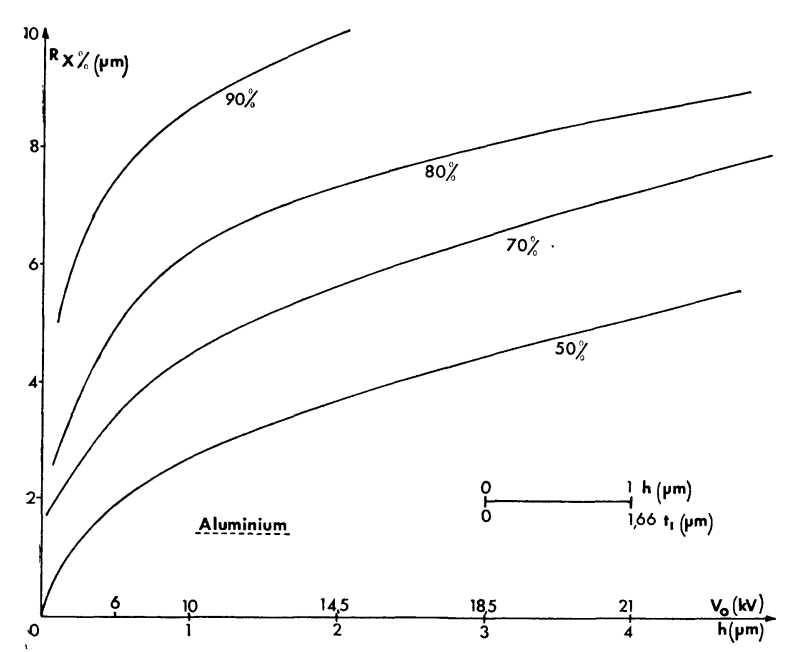

(a)

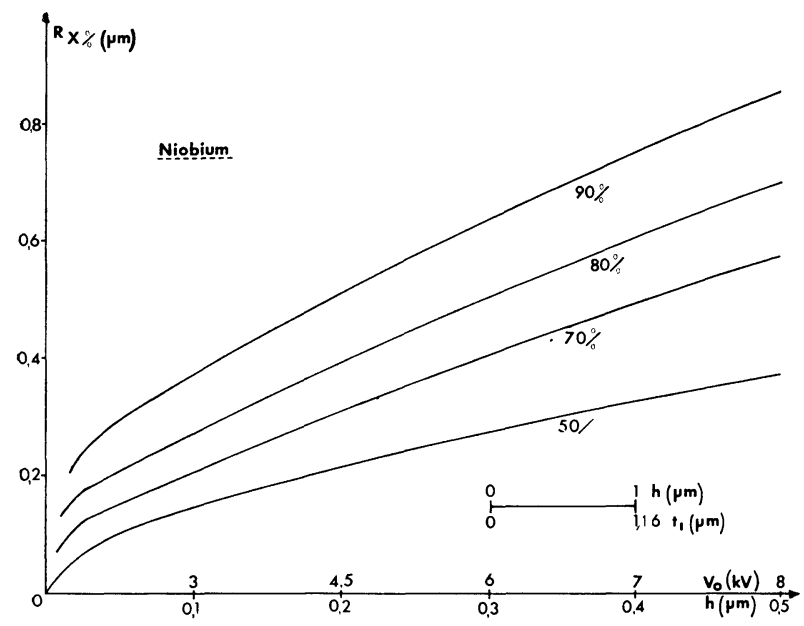

(b)

Fis. 8. - Evolution du rayon des aires circulaires émettant respectivement $50 \%, 70 \%$ et $80 \%$ de l'intensité photoélectronique détectée (soit $R_{50 \%}, R_{70} \%, R_{80 \%}$ ) en fonction de la distance de la source $\mathrm{X}$ à l'objet (h) et de la tension d'accélération optimale $V_{0}$ des électrons $a$ ) Anticathode en Aluminium. b) Anticathode en Niobium.
On peut constater que pour une anticathode en aluminium caractérisée par un paramètre « $h$ » égal à $2 \mu \mathrm{m}\left(V_{0}=14,5 \mathrm{kV}, t_{1}=3,3 \mu \mathrm{m}\right) 50 \%$ de l'intensité photoélectronique détectée (soit environ $10^{6} \mathrm{e} / \mathrm{s}$ ) sera émise par une tache circulaire de rayon $R_{50 \%} \simeq 3,75 \mu \mathrm{m}, 80 \%$ de cette intensité sera émise par une tache circulaire de rayon $R_{80 \%} \simeq 7,5 \mu \mathrm{m}$ et $90 \%$ de l'intensité avec $R_{90 \%} \simeq 10 \mu \mathrm{m}$.

Avec le même type d'anticathode mais en choisissant $h$ sensiblement égal à $0,5 \mu \mathrm{m}\left(V_{0} \simeq 6 \mathrm{kV}\right)$ les valeurs successives de $R$ deviennent $R_{50 \%} \simeq 1,8 \mu \mathrm{m}$, $R_{80 \%} \simeq 5 \mu \mathrm{m}, R_{90 \%} \simeq 7,5 \mu \mathrm{m}$. L'emploi des radiations du type $M_{\xi}$ se révèle ici être intéressant car, comme le matériau constituant l'anticathode est plus lourd, on peut pour tension d'accélération $V_{0}$ donnée, utiliser des anticathodes plus minces qu'avec l'aluminium. Pour une anticathode en niobium, par exemple $h$ est égal à $0,25 \mu \mathrm{m}$ pour $V_{0} \simeq 5 \mathrm{kV}$ et les valeurs correspondantes de $R$ sont $R_{50 \%} \simeq 0,25 \mu \mathrm{m}$, $R_{80 \%} \simeq 0,45 \mu \mathrm{m}, R_{90 \%} \simeq 0,55 \mu \mathrm{m}$.

Le changement d'anticathode réduit donc de façon appréciable les dimensions de la tache émissive tout en maintenant l'intensité du signal détecté à un niveau appréciable $\left(3 \times 10^{3} \mathrm{cps} / \mathrm{s}\right.$, Fig. $\left.6 b\right)$.

$\mathrm{Si}$ on définit arbitrairement la résolution spatiale du système « $\delta$ » comme étant le diamètre de la tache susceptible d'émettre $80 \%$ de l'intensité photoélectronique totale on peut, compte tenu des tableaux I et III et des courbes précédentes, établir le tableau IV qui résume les principaux résultats.

On constate donc qu'une résolution de l'ordre de $1 \mu \mathrm{m}$ peut être atteinte par l'emploi d'anticathode en niobium tout en conservant une intensité photoélectronique $N(\theta, \Delta \theta)$ aisément mesurable $\left(10^{4} \mathrm{c} / \mathrm{s}\right)$ mais à côté de cet intérêt évident, nous devons signaler que, en contrepartie, les éléments tels que $\mathrm{C}, \mathrm{N}$ et $\mathrm{Cl}$ ne pourront être mis en évidence car ils n'ont pas de souscouche électronique susceptible d'être excitée par une telle radiation $\left(20<E_{\mathrm{L}}<170 \mathrm{eV}\right)$. Comme pour la microsonde électronique, une amélioration de la réso-

\section{TABLEAU IV}

Evolution de l'intensité photoélectronique détectée $N(\theta, \Delta \theta)$ en fonction de la "résolution spatiale 》 désirée « $\delta »$ ( $\delta$ représente le diamètre de la surface circulaire qui émet $80 \%$ de l'intensité photoélectronique totale).

On précise en outre la valeur à conférer aux paramètres ajustables (tension $V_{0}$, intensité $I_{0}$ diamètre $d_{0}$ de la sonde électronique incidente, nature et épaisseur $t_{1}$ de l'anticathode).

$$
\delta
$$

\begin{tabular}{|c|c|c|c|c|c|c|c|c|}
\hline $\begin{array}{l}2 R 80 \% \\
\quad(\mu \mathrm{m})\end{array}$ & $\begin{array}{c}R 50 \% \\
(\mu \mathrm{m})\end{array}$ & $\begin{array}{l}\text { anti- } \\
\text { cathode }\end{array}$ & $\begin{array}{c}N(\theta, \Delta \theta) \\
\mathrm{e} / \mathrm{s}\end{array}$ & $\begin{array}{c}V_{0} \\
(\mathrm{kV})\end{array}$ & $\begin{array}{c}h \\
(\mu \mathrm{m})\end{array}$ & $\begin{array}{c}t_{1} \\
(\mu \mathrm{m})\end{array}$ & $\begin{array}{c}d_{0} \\
(\mu \mathrm{m})\end{array}$ & $\begin{array}{c}I_{0} \\
(\mu \mathrm{A})\end{array}$ \\
\hline - & - & 一 & - & - & - & - & - & - \\
\hline 15 & 4 & $\mathrm{Al}$ & $2 \times 10^{6}$ & 14,5 & 2 & 3,2 & 4 & 9 \\
\hline 10 & 1,75 & $\mathrm{Al}$ & $8 \times 10^{3}$ & 6,5 & 0,5 & 0,8 & 1 & 0,1 \\
\hline 4 & & $\mathrm{Nb}$ & $2 \times 10^{4}$ & 13,2 & 1,25 & 1,5 & 2,5 & 2,4 \\
\hline 1 & 0,27 & $\mathrm{Nb}$ & $1 \times 10^{4}$ & 6 & 0,3 & 0,35 & 0,7 & 0,045 \\
\hline 0,75 & 0,2 & $\mathrm{Nb}$ & $2 \times 10^{3}$ & 4,5 & 0,2 & 0,24 & 0,35 & 0,01 \\
\hline
\end{tabular}


lution peut être obtenue par l'emploi de tensions plus faibles (et ici d'anticathodes plus minces) mais ce gain en résolution entraîne parfois une diminution dramatique de l'intensité des signaux émis. Signalons toutefois que, dans ce domaine énergétique, c'est la limite électronique (et non la limite thermique) qui conditionne directement l'intensité $I_{0}$ reçue par l'anticathode et que cette intensité peut être accrue par l'emploi de cathodes en hexaborure de lanthane (Loeffler [18]) ou de cathodes à pointe (Mulvey [23]) à défaut de l'utilisation de canons à émission (ils ne se justifieraient que pour obtenir des sondes de plus petit diamètre) (Veneklasen [24]) l'amélioration d'une puissance de $10 \mathrm{du}$ coefficient numérique placé dans l'expression (16) se répercuterait de façon identique sur l'intensité photoélectronique.

D'autre part, comme l'analyseur de Citrin et coll. tolère une source objet ayant un diamètre $" a$ » de $1 \mathrm{~mm}$, l'intensité évaluée dans le tableau IV pourra être effectivement détectée en fonctionnement en microanalyseur (même avec une résolution de $15 \mu \mathrm{m}$ ) et en fonctionnement à balayage, le nombre de points par ligne correspondra sensiblement à $a / \delta$ soit environ 1000 points par ligne (et 1000 lignes) si les conditions pour obtenir une résolution de $1 \mu \mathrm{m}$ sont satisfaites.

4.2 REMARQUES CONCERNANT LA RÉSOLUTION SPATIALE. - 4.2.1 Inconvénients liés à l'extension latérale des faisceaux photoélectroniques. - Se référant à un échantillon de composition homogène et d'épaisseur supposée constante sur toute la surface de l'anticathode, la définition de la résolution donnée plus haut ( $\delta \simeq 2 R_{80} \%$ ) procède d'un choix purement arbitraire ; il serait possible de l'améliorer artificiellement en choisissant un pourcentage moindre (par exemple $70 \%$ voire $60 \%$ ou au contraire s'inquiéter de ce que $20 \%$ du signal reçu puissent venir d'une partie de l'échantillon extérieure à la tache de diamètre $" \delta$ » mais, s'il s'agit de déceler à la surface de l'anticathode un élément n'ayant qu'une seule concentration possible, on observera que ce pourcentage d'intensité ne sera atteint que si cet élément occupe toute la surface entourant l'aire analysée (Fig. 9a) alors que la contri-
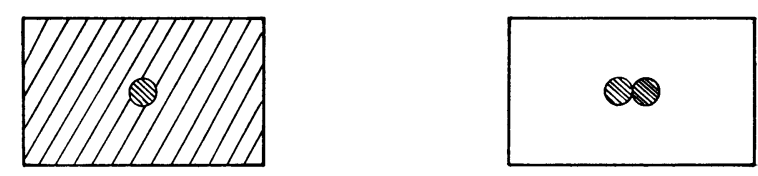

FIG. 9. - Voir texte.

bution supplémentaire parasite due à la présence d'une source émissive de même concentration et de mêmes dimensions juxtaposée à la zone analysée (Fig. 9b) ne dépassera pas $3 \%$ de l'intensité mesurée.

En fait la situation la plus défavorable concerne le cas où 2 sources émissives juxtaposées ont des concentrations nettement différentes de telle manière que dans l'examen de la zone ayant la concentration la plus faible, le signal parasite venant de la zone de plus forte concentration soit prépondérant.

Aussi, à défaut d'autres solutions, il faudra prévoir en microsonde électronique des corrections numériques de l'intensité émise par la zone analysée à partir des intensités mesurées pour tous les points entourant cette zone.

En microscopie à balayage, les différentes opérations (stockage de l'image initiale et corrections d'intensité) pourraient être pilotées par ordinateur de façon à ce que l'image finale restituée sur tube de télévision comporte plusieurs tons de gris. A défaut d'un tel traitement de l'information, il sera au moins possible d'obtenir une image binaire en fixant un seuil de détection du courant photoélectronique légèrement supérieur au seuil imposé par le bruit de fond habituel de manière à éliminer artificiellement la contribution des signaux parasites ; la qualité de l'image finale sera alors comparable à celle obtenue avec le microscope à balayage reposant sur l'effet Auger (MacDonald et Waldrop [25], Griffiths et Wardell [26]).

Remarque: L'influence de la réflexion et de la réfraction des rayons $X$ à l'interface anticathode objet, influence que nous n'avons pas prise en compte dans l'évaluation de l'intensité photoélectronique globale (15), est certainement négligeable en valeur absolue compte tenu du fait que, aux longueurs d'ondes considérées, les indices optiques complexes $(\tilde{N}=n+i k)$ des matériaux ont une partie réelle très voisine de l'unité et une partie imaginaire très faible, ce qui entraîne un coefficient de réflexion quasiment nul et un transfert quasi intégral du flux $\mathrm{X}$ de l'anticathode vers l'objet. Néanmoins, pour des angles $\Phi$ et $\Phi^{\prime}$ dont la valeur (faible) croîtra avec la longueur d'ondes une pseudo-réflexion totale peut se produire à cette interface et éliminer une partie de l'intensité photoélectronique parasite : c'est ainsi que Henke [13] a montré, en étudiant ce phénomène, que $50 \%$ de l'intensité $X$ était réfléchie, à l'interface vide-or, pour un angle $\Phi$ qui passait de $25 \mathrm{mrad}\left(\operatorname{avec} \mathrm{AlK}_{\alpha}\right)$ à $230 \mathrm{mrad}$ pour $\mathrm{BeK}_{\alpha}$ $(109 \mathrm{eV})$.

Etant donné le manque d'informations relatives à la valeur de certaines constantes optiques (essentiellement celles du niobium à $170 \mathrm{eV}$ ) il ne nous a pas été possible d'estimer, à partir des formules de Fresnel généralisées, l'amélioration qui serait apportée à la résolution par la prise en compte de cet effet ; ultérieurement il ne sera pas exclu d'améliorer la résolution par l'interposition entre l'anticathode et l'objet d'un film mince judicieusement choisi.

4.2.2 Limitation géométrique de l'aire émissive. Pour éviter de tels inconvénients, on peut appliquer contre l'objet des diaphragmes qui limiteront géométriquement l'aire émissive (Fig. 10a), il sera alors possible d'utiliser une anticathode en aluminium et un diaphragme d'un micron pour atteindre une résolution spatiale de cet ordre de grandeur (l'intensité chutant 
de $2 \times 10^{6} \mathrm{e} / \mathrm{s}$ à $10^{4} \mathrm{e} / \mathrm{s}$ ) mais, si un tel système peut être appliqué en microanalyse locale, il est difficile à concevoir en microscopie photoélectronique à balayage car il nécessiterait le déplacement du diaphragme en synchronisme avec le balayage électronique (ou bien - ce qui revient au même - il faudrait assurer le balayage par le seul déplacement mécanique de l'échantillon).

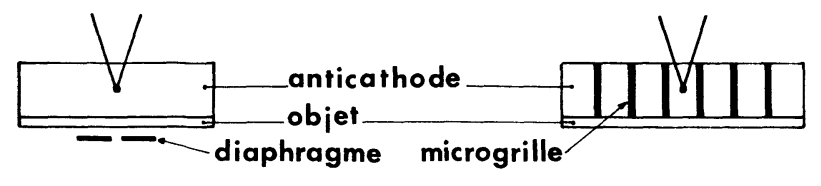

$\mathbf{a}$

b

FIG. 10. - Limitation géométrique de la résolution : $a$ ) avec un diaphragme; $b$ ) en immergeant l'anticathode dans une microgrille découpée dans un métal fortement absorbant.

Il semble donc préférable d'immerger dans l'anticathode une microgrille métallique qui atténuera presque totalement la diffusion latérale des rayons $\mathrm{X}$ (voir Fig. b) si le métal ( $\mathrm{Pt}, \mathrm{Cu}, \mathrm{W})$ de la microgrille les absorbe fortement. Ce système, qui peut être aisément obtenu par vaporisation sous vide de l'anticathode sur la microgrille, autorise aussi bien en microscopie qu'en microanalyse une résolution spatiale limitée par les dimensions des mailles de grilles utilisées [si, à notre connaissance les meilleures grilles actuellement disponibles dans le commerce ont un pas de $12,5 \mu \mathrm{m}$ ( $2000 \mathrm{mesh}$ ), des plaques métalliques percées de trous plus petits sont parfaitement concevables].

4.3 ANAlyse QUANTITATIVE ET SENSIBILITÉ DE LA MÉTHODE. - En marquant une monocouche d'acide stéarique avec de l'iode, Siegbahn et ses collaborateurs [1] ont montré qu'il était possible de déceler $10^{-8} \mathrm{~g}$ de l'halogène (soit une sensibilité superficielle de $10^{15} \mathrm{at} / \mathrm{cm}^{2}$ ). Mis à part cette tentative initiale très peu d'études (Ebel [27]) ont été, jusqu'ici, consacrées à l'analyse quantitative par spectroscopie des photoélectrons; quand les sections efficaces partielles de photoionisation seront mieux connues, il sera possible de les entreprendre, au moins, sur des surfaces planes homogènes et exemptes de contamination. Dans cette perspective signalons simplement que l'expression (11) permet de déduire de mesures absolues d'intensité la concentration des différents éléments constituant une couche superficielle (d'épaisseur $t_{0}$ plus grande que $\lambda_{\mathrm{e}}$ ) et supposée homogène sur une étendue de l'ordre de grandeur de la résolution spatiale (si l'épaisseur de cette couche superficielle est inférieure à $\lambda_{\mathrm{e}}$, l'intégration (sur $t$ ) de la formule (8) fait apparaître un facteur $\exp -\frac{t}{\lambda_{\mathrm{e}} \cos \theta}$ qui permettra, comme dans les montages plus classiques (Fadley et coll.), de déterminer cette épaisseur à partir des mesures angulaires $I=f(\theta))$. Outre la précision de la méthode, il est important, aussi, de connaître sa sensibilité ultime; c'est ainsi que, à partir de considérations sur le rapport signal sur bruit, nous développons ces études dans le chapitre suivant afin de pouvoir les comparer avec les performances que donne la spectrométrie Auger.

5. Comparaison entre la technique proposée et la spectrométrie Auger. - On oppose souvent la spectrométrie photoélectronique $\mathrm{X}$ à la spectrométrie Auger et d'excellents articles de synthèse [28], [29], [30] ont été consacrés à ce sujet. Nous ne souhaitons pas reprendre ici les arguments amplement développés dans ces articles mais préciser de façon plus quantitative quelles sont les comparaisons que l'on peut formuler entre la spectrométrie Auger et l'analyse photoélectronique $\mathrm{X}$ qui serait effectuée à partir du montage que nous préconisons ; dans ce dernier cas, cela nous permettra de résumer de façon plus claire les performances que l'on peut en attendre et de mentionner des données numériques qui auraient été omises (par exemple : rapport signal sur bruit, limite de sensibilité, etc...).

5.1 EPAISSEUR DE LA ZONE ANALYSÉE. - L'épaisseur de la zone analysée est donnée directement par la profondeur d'extraction des photoélectrons $\lambda_{\mathrm{e}}$ ou des électrons Auger $\lambda_{\mathrm{A}}$. Elle caractérise le libre parcours moyen élastique des électrons correspondants et dépend essentiellement de leur énergie cinétique et de façon moins nette de la nature du milieu dans lequel ils se propagent (voir par exemple l'article de Powell et les références citées [31]). Comme l'énergie cinétique des électrons dans les deux techniques est sensiblement comparable $\left(E_{\mathrm{c}}<1000 \mathrm{eV}\right)$ nous admettrons que la profondeur de la zone analysée est la même (5-50 $\AA$ ) bien que l'analyse Auger par excitation électronique rasante puisse plus privilégier la surface que la spectrométrie photoélectronique $\mathrm{X}$.

5.2 RENDEMENT QUANTIQUE GLOBAL DU DISPOSITIF PRÉCONISÉ ET DE L'EFFET AUGER. - Si, dans l'expression (11), on remplace $Q_{0}$ par $I_{0} \eta_{\mathrm{x}}$ (dans laquelle $\eta_{\mathrm{x}}$ représente le rendement de l'émission $X$ dans l'anticathode tel qu'on peut le déduire de l'expression (15)), on obtient le rendement quantique global c'est-à-dire le nombre de photoélectrons détectés par électron primaire frappant l'anticathode soit :

$$
\begin{aligned}
\eta_{\mathrm{T}}=\frac{I_{\mathrm{pe}}}{I_{0}}=\left[\frac{1}{2} \eta_{X}\right]\left[\rho \sigma_{\mathrm{nl}}\right. & \left.\lambda_{\mathrm{e}} \cos \theta\right] \times \\
& \times\left[\frac{\sin \theta \Delta \theta}{2}\right]\left[E_{1}(\mu h)\right] .
\end{aligned}
$$

On constate, en se reportant aux tableaux I, II et III, que ce rendement global est typiquement de l'ordre de $10^{-8}$ (rapport entre la dernière colonne $I_{\mathrm{pe}}$ et la 9e colonne $\left(I_{0}\right)$ ).

- Pour évaluer l'intensité Auger $I_{\mathrm{A}}$ détectée par l'analyseur miroir cylindrique et issue d'un échantillon homogène bombardé à incidence normale par un faisceau primaire $I_{0}$, on doit théoriquement prendre en compte d'une part, l'affaiblissement en énergie et en 
intensité du faisceau incident quand il progresse dans l'échantillon et d'autre part, l'effet Auger provoqué par les électrons secondaires et par les électrons primaires rétrodiffusés (Bauer [28], Todd [29], Brundle [30]) ; si, pour fixer les idées, on admet que ces deux effets se compensent plus ou moins, il est alors possible d'obtenir l'équivalent de la formule (11) pour l'effet Auger (avec pour les électrons Auger, les approximations déjà formulées pour les photoélectrons aux $\S a, b, d, f, h$ du chapitre 3.3).

On obtient alors l'expression (19) :

$I_{\mathrm{A}}(\theta, \Delta \theta)=\left[I_{0}\right]\left[\rho Q_{\mathrm{i}} a_{\mathrm{ijk}} \lambda_{\mathrm{A}} \cos \theta\right]\left[\frac{\sin \theta \cdot \Delta \theta}{2}\right]$

dans laquelle $Q_{\mathrm{i}}$ représente la section efficace d'ionisation de la (sous) couche $i$ et $a_{\mathrm{ijk}}$ la probabilité pour qu'un électron effectue une transition Auger ou CosterKronig, d'une (sous) couche $\mathrm{j}$ vers la (sous) couche $\mathrm{i}$ avec émission d'un électron Auger issue de la (sous) couche $\mathrm{k}$.

On retrouve ( $3^{e}$ terme) le coefficient de transmission de l'analyseur et le deuxième terme (homologue du $2^{\mathrm{e}}$ terme de l'expression (11)) traduit le rendement quantique de l'effet Auger proprement dit (avec $\lambda_{\mathrm{A}}$ le libre parcours moyen élastique des électrons Auger).

Appliquée au carbone et à l'aluminium, en considérant uniquement l'ionisation de la couche $K$ avec les valeurs numériques de $Q_{\mathrm{imax}}$ rapportées par Bauer [28], on peut estimer le rendement quantique $\eta_{\mathrm{A}}\left(=I_{\mathrm{A}} / I_{0}\right)$ de l'effet Auger pour ces deux éléments et on obtient :

- Carbone $: \eta_{\mathrm{A}} \simeq 2 \times 10^{-4}$.

avec

$$
\begin{aligned}
&\left(Q_{\mathrm{i}} \simeq 3,5 \times 10^{-19} \mathrm{~cm}^{2}[28], a_{\mathrm{ijk}} \simeq 1,\right. \\
&\left.\lambda_{\mathrm{A}} \simeq 25 \AA, \frac{\sin \theta \Delta \theta}{2} \simeq 4 \times 10^{-2}\right) .
\end{aligned}
$$

Aluminium $: \eta_{\mathrm{A}} \simeq 10^{-6}$

avec

$$
\begin{aligned}
&\left(Q_{\mathrm{i}} \simeq 10^{-20} \mathrm{~cm}^{2}[28], a_{\mathrm{ijk}}\right. \simeq 1, \\
&\left.\lambda_{\mathrm{A}} \simeq 25 \AA, \frac{\sin \theta \Delta \theta}{2} \simeq 0,85 \times 10^{-2}\right),
\end{aligned}
$$

alors que Chang [32] évalue à $10^{-5}$ l'ordre de grandeur du rapport expérimental $I_{\mathrm{A}} / I_{0}$ (sans préciser toutefois ni la nature de l'élément étudié ni le type d'analyseur employé : CMA ou LEED-Auger).

5.3 EVOLUTION DES INTENSITÉS EN FONCTION DE LA NATURE DES ÉLÉMENTS EXAMINÉS. - Il est bien connu que l'effet Auger est d'autant plus intense que l'élément examiné à un numéro atomique $Z$ faible et les deux exemples numériques indiqués ci-dessus illustrent bien ce propos ; quantitativement la section efficace maximale d'ionisation $Q_{\mathbf{i}}$ obéit sensiblement [28] à la loi :

$$
Q_{\mathrm{imax}} \approx \frac{250}{E_{\mathrm{i}}^{2}}\left[\AA^{2}\right]
$$

et sera donc d'autant plus importante que l'énergie d'ionisation $E_{\mathbf{i}}(\mathrm{eV})$ de la couche considérée sera faible.

Il sera donc toujours possible de tempérer cet effet en ionisant, pour les éléments de $Z$ élevé, les niveaux des moins liés ; mais ces niveaux initiaux, étant plus nombreux cela peut conduire à une redistribution de l'intensité Auger entre les raies associées aux multiples transitions possibles (avec une compétition entre les différentes probabilités $a_{\mathbf{i j k}}$ et avec, dans le cas par exemple, de l'aluminium une largeur énergique des raies LVV conditionnée par l'énergie de Fermi de corps $\simeq 12 \mathrm{eV}$ voir plus loin).

Inversement, la section efficace d'atténuation des photons $\mathrm{X}$ obéit approximativement à la relation empirique (voir réf. [1] p. 142) :

$$
\mu \approx c Z^{4} \Lambda_{\mathrm{x}}^{3}+b
$$

Comme l'absorption des rayons $\mathrm{X}$ est essentiellement due (aux longueurs d'onde considérées) à l'effet photoélectronique, on pourrait s'attendre à ce que les raies photoélectroniques des éléments lourds soient beaucoup plus intenses que les autres mais on doit considérer que la section efficace totale se redistribue en sections efficaces partielles $\sigma_{\mathrm{n} 1}$ de plus en plus nombreuses au fur et à mesure que $Z$ croît. Comme en outre ces sections efficaces partielles ont un poids relatif qui varie en fonction de $\Lambda_{\mathrm{x}}$ [19] il est très difficile d'établir actuellement une loi rigoureuse de l'évolution de l'intensité photoélectronique $X$ relative à une souscouche donnée en fonction de $Z$. (C'est d'ailleurs dans ce sens que les applications numériques des tableaux $I$, II et III ne sont mentionnées que pour fixer des ordres de grandeurs). En conséquence, si on peut affirmer que la spectrométrie Auger donne des signaux plus intenses pour les éléments légers que pour les éléments lourds alors que la spectrométrie photoélectronique $\mathrm{X}$ donne des résultats inverses, il est difficile de dépasser ce stade qualitatif sans un examen élément par élément qui serait hors du but assigné à cet article (cela supposerait d'ailleurs une meilleure connaissance des sections efficaces dont on trouvera néanmoins quelques exemples dans la référence [30]).

5.4 RetOUR SUR LE RAPPORT ENTRE $I_{\mathrm{A}}$ ET $I_{\mathrm{pe}}$ - $-\mathrm{A}$ partir des relations (18) et (19) et pour prolonger la comparaison entre les deux techniques on peut évaluer le rapport entre les intensités détectées : photoélectronique et Auger (avec une excitation à incidence normale). Si ces intensités sont relatives à un même échantillon et si elles sont mesurées à l'aide d'analyseurs ayant des performances comparables (comme par exemple dans le dispositif représenté sur la Fig. 1 avec le canon à électrons annexe (9), on obtient (en admettant, en outre, que l'intensité primaire est la même dans les deux cas) :

$$
\frac{I_{\mathrm{pe} .}(\theta, \Delta \theta)}{I_{\mathrm{A}}(\theta, \Delta \theta)} \approx \frac{1}{2} \eta_{\mathrm{x}} E_{1}(\mu h) \cdot \frac{\sigma_{\mathrm{nl}}}{a_{\mathrm{ijk}} \cdot Q_{\mathrm{i}}} .
$$


On constate donc que le coefficient $10^{3}$ privilégiant l'intensité Auger vis-à-vis de l'intensité photoélectronique provient, à sections efficaces $\left(\sigma_{\mathrm{n} 1}\right.$ et $Q_{\mathrm{i}}$ supposées comparables [30] du rendement quantique $\eta_{\mathrm{x}}$ relatif à la production des rayons $X$ dans l'anticathode. Toutefois on va voir que le déficit d'intensité correspondant est compensé par le fait que d'une part les raies Auger s'inscrivent sur un fond continu qui est très intense, ce qui impose le plus souvent l'emploi de techniques de différenciation en courant des courbes $N(E)$ pour repérer leur position alors que, d'autre part, les raies photoélectroniques émergent nettement $\mathrm{du}$ fond continu des électrons inélastiques et peuvent être comptées en impulsions.

5.5 RAPPORT SIGNAL SUR BRUIT ET LIMITE DE SENSIBILITÉ. - Chang [32] évalue le courant de bruit se référant à l'effet de grenaille (shot effect) qui limite la sensibilité de la spectrométrie Auger. A partir de la formule de Schottky $-\Delta I=\sqrt{2 \cdot e \cdot I \cdot \Delta f}-(23)$ - et par l'emploi d'un analyseur miroir cylindrique, il obtient une limite de sensibilité (rapport signal sur bruit $=1$ ) de $10^{10} \mathrm{at} / \mathrm{cm}^{2}$ pour les impuretés de surface soit $2 \times 10^{16} \mathrm{at} / \mathrm{cm}^{3}$ pour les impuretés de volume.

- En spectrométrie des photoélectrons $X$ et par comptage d'impulsions, le phénomène d'émission aléatoire d'électrons conduit à un rapport signal sur bruit donné par la statistique de Poisson, soit :

$$
\frac{\text { Signal }}{\text { Bruit }}=\sqrt{N}
$$

dans laquelle $N$ représente le nombre d'électrons détectés pendant le temps $t$.

Or, le temps de comptage d'une raie photoélectronique peut atteindre $100 \mathrm{~s}$ (on trouvera des exemples fréquents sur les spectres expérimentaux publiés dans la référence [1] et on observera qu'une raie photoélectronique dont l'intensité est de $1 \mathrm{cps} / \mathrm{s}$ est parfaitement décelable puisque le rapport signal sur bruit sera de 10 si $t=100 \mathrm{~s})$.

Donc, comme nous obtenons une intensité prévisible de l'ordre de $10^{4} \mathrm{cps} / \mathrm{s}$ pour un film homogène d'or $\left(\rho=6 \times 10^{22} \mathrm{at} / \mathrm{cm}^{3}\right)$ déposé sur une anticathode en Niobium, cela nous conduit (avec le même temps de comptage de $100 \mathrm{~s}$ ) à une sensibilité théorique limite de $6 \times 10^{16} \mathrm{at} / \mathrm{cm}^{3}$ pour le dispositif que nous préconisons; avec $\lambda_{\mathrm{e}} \simeq 25 \AA$, cela correspond aussi à une sensibilité superficielle de $1,5 \times 10^{10} \mathrm{at} / \mathrm{cm}^{2}$.

Il est évident que cette limite théorique pourrait être améliorée par l'emploi de l'anticathode en aluminium $\left(10^{6} \mathrm{cps} / \mathrm{s}\right.$ au lieu de $10^{4} \mathrm{cps} / \mathrm{s}$ pour une anticathode en $\mathrm{Nb}$, mais au détriment de la résolution spatiale) ou par un temps de comptage plus long; toutefois, dans cette dernière hypothèse et comme le note Chang [32] pour l'effet Auger, il faut remarquer que quand on atteint de telles sensibilités, la détection limite doit être établie par d'autres considérations car les propretés de surface correspondantes ne peuvent être maintenues plus de quelques minutes dans un vide de $10^{-10}$ torr.
5.6 Microanalyse Quantitative. - Quand les sections efficaces partielles de photoionisation seront mieux connues, il est parfaitement concevable d'utiliser, en spectrométrie des photoélectrons, la formule (11) pour faire de la microanalyse quantitative c'est-à-dire pour pouvoir donner la concentration respective de 2 éléments à partir de l'intensité des raies photoélectroniques correspondantes.

- Un tel projet est beaucoup plus délicat en spectrométrie Auger car indépendamment du fait que le signal exploitable est souvent le signal dérivé, il est difficile de tenir compte des effets de rétrodiffusion (il est hors de question d'utiliser la formule (19) qui n'a été donnée que pour simplifier les comparaisons); en outre, comme le signale Brundle [30], si le processus Auger concerne un niveau électronique situé dans la bande de valence, la raie Auger correspondante (intensité et profil) relative à un élément devient très sensible à son environnement chimique ce qui rendra nécessairement la microanalyse Auger plus approximative.

5.7 Résolutions SPATIALES ET MICRoscopies A BalaYaGe. - En microscopie et microanalyse Auger, la résolution spatiale est donnée par les dimensions latérales des volumes immergés par les électrons incidents et correspond, en première approximation, au diamètre de la sonde électronique utilisée; dans le système étudié ici ce sont les dimensions de l'aire éclairée par les rayons $X$ émis par la sonde électronique qui caractérisent cette résolution. Il ne fait donc pas de doute que cette dernière aire sera toujours plus grande que la source qui lui a donné naissance. On peut donc estimer que la résolution dans notre dispositif sera plus mauvaise que dans le dispositif Auger mais on peut observer que cette détérioration peut être limitée à un coefficient de l'ordre de 2 (ou moins) par le choix d'une anticathode en métal lourd comme le niobium : pour une résolution de $1 \mu \mathrm{m}$ par exemple le diamètre de la sonde $d_{0}$ est de $0,7 \mu \mathrm{m}$.

Dans le mode à balayage, les performances prévisibles du microscope photoélectronique peuvent être déduites du tableau IV, compte tenu de la définition habituelle du contraste $C$ de ce mode [33] [34] $\left(N \geqslant \frac{1}{C^{2}} \cdot 100\right)$

Avec une résolution spatiale de $15 \mu \mathrm{m}$, il sera possible d'obtenir en $100 \mathrm{~s}$ une image de 100 lignes avec 100 points par ligne et comportant plusieurs tons de gris $(C \simeq 10 \%)$; si on cherche à améliorer la résolution spatiale, on retombe sur le compromis habituel entre celle-ci et le contraste puisque pour la même durée de lecture $(100 \mathrm{~s})$ et avec la même définition $(100 \times 100)$, une résolution spatiale de $1 \mu \mathrm{m}$ ne permet de visualiser qu'une image binaire $(C \simeq 100 \%)$.

On peut faire varier les différents paramètres et, en accroissant le temps d'enregistrement, il sera possible d'améliorer le contraste ou la définition de l'image mais les performances attendues doivent être du même ordre de grandeur que celles obtenues par MacDonald et 
Waldrop en microscopie Auger [25] compte tenu du fait que, dans cette dernière technique, le temps de lecture de l'objet est accru par la nécessité de différencier le signal Auger émis point par point.

5.8 INTERPRÉTATION DES SPECTRES ET GLISSEMENT CHIMIQUE. - L'ESCA ne met en jeu qu'un niveau atomique : les spectres sont donc faciles à interpréter, la confusion entre les éléments est peu probable et le glissement chimique est aisément identifiable.

- Dans le processus Auger, la largeur des raies n'est pas limitée par la largeur énergétique de la source et il est donc théoriquement possible d'enregistrer ces raies avec une résolution de $0,2 \mathrm{eV}$; en pratique elles sont beaucoup plus larges qu'en ESCA parce que l'effet Auger met en jeu 3 niveaux atomiques et que la bande de valence se trouve être fréquemment concernée (dans les cas extrêmes la largeur du pic Auger peut atteindre deux fois la largeur de la bande de valence dans le cas où la raie étudiée est du type XVV).

En conséquence, outre la multiplicité des raies Auger (et les artefacts possibles liés à la différenciation du signal) qui compliquent l'identification des éléments, la mise en évidence du glissement chimique et son exploitation seront souvent sujet à caution en spectrométrie Auger - on en trouvera des exemples abondants dans la référence [30].

\subsection{EFFETS DU FAISCEAU SUR LA SURFACE ÉTUdIÉE. -} L'interaction des faisceaux incidents avec la surface peut modifier celle-ci et, dans ce domaine, les électrons semblent causer plus de problèmes que les photons $X$. Outre les effets créés d'élévation de température (et dont notre projet n'est pas toujours exempt), on peut citer [30] : la désorption et la diffusion provoquées par les électrons incidents, la dissociation des espèces absorbées, la décomposition du support et la contamination de la surface.

Ces effets peuvent être minimisés en réduisant l'intensité de la sonde électronique mais il n'en reste pas moins que le rayonnement $X$ est beaucoup moins perturbateur que le bombardement électronique et que certaines études ne pourront être menées à leurs termes que par l'emploi de la spectroscopie photoélectronique $X$ (on trouvera dans la référence [35] un exemple montrant comment un problème métallurgique a pu être résolu en XPS avec une résolution spatiale de $1 \mathrm{~mm}$ alors que la spectroscopie Auger s'était avérée impuissante malgré une résolution au moins 100 fois meilleure).

5.10 INCONVÉNIENTS SPÉCIFIQUES A NOTRE PROJET ET AMÉLIORATIONS POSSIBLES. - a) Inconvénients. Pour conférer à la spectroscopie des photoélectrons un caractère microanalytique et autoriser la formation d'images spécifiques des éléments constituant la surface d'un échantillon, la technique que nous proposons impose deux contraintes qui n'ont pas d'équivalent en spectrométrie Auger :

- l'échantillon doit avoir la forme d'un film mince,
- l'intensité optimale pour une résolution spatiale donnée ne peut être obtenue que par un changement d'anticathode.

Ces inconvénients sont réels toutefois, comme les techniques comparées ici ont une très grande sensibilité superficielle, elles imposent d'autres contraintes concernant la propreté de la surface et la nécessité d'opérer dans un vide élevé qui font qu'elles sont surtout réservées à l'étude de mécanismes fondamentaux sur les surfaces - à défaut de leur utilisation en contrôle industriel - ce qui minimise sans les supprimer les inconvénients cités plus haut.

b) Améliorations possibles. - Nous avons présenté, dans le présent article, les performances prévisibles concernant un appareil de conception originale. Comme les résultats étaient obtenus sur le papier, nous avons essayé de formuler des estimations raisonnables sans nécessairement faire appel aux derniers raffinements de la technique.

C'est ainsi que le faisceau électronique incident est délivré par une cathode classique en tungstène à défaut de l'emploi de cathode en $\mathrm{LaB}_{6}$ (ou à émission de champ) ce qui aurait nécessairement amélioré l'intensité détectée, la résolution spatiale, etc... Nous aurions pu, aussi, tenir compte du fait que dans le mode à balayage l'échauffement réel de l'anticathode sera plus faible que celui qui a été calculé car l'emploi du balayage est alors équivalent à l'emploi d'une anticathode tournante et l'accroissement de la charge électronique sur l'anticathode accroîtra alors les caractéristiques de l'image finale (définition, contraste, etc...).

Enfin l'utilisation des radiations du type $\mathbf{M}_{\xi}$ est relativement récente et la mise en œuvre probable de nouvelles radiations caractéristiques dans la gamme des $500 \mathrm{eV}$, à défaut de l'utilisation de radiations $\mathrm{X}$ non conventionnelles, rendra possible la microanalyse $\mathrm{du}$ carbone avec une résolution spatiale meilleure que $10 \mu \mathrm{m}$ et une résolution énergétique de l'ordre de $1 \mathrm{eV}$ (en effet actuellement le carbone peut être décelé par les radiations allant de $\mathrm{T}_{\mathrm{i}} \mathrm{L}_{1}$ à $\mathrm{AlK}_{\alpha}$ - dans le tableau I de la référence [19] - mais dans des conditions telles que ou $\Delta E>1 \mathrm{eV}$ ou $\delta>1 \mu \mathrm{m}$ ).

5.11 CONCLUSIONS CONCERNANT LA COMPARAISON ENTRE LES DEUX TECHNIQUES. - Des comparaisons détaillées effectuées ci-dessus, il ressort que chaque technique possède des caractères qui lui sont spécifiques ; en effet, si la profondeur d'extraction et la sensibilité sont comparables, l'intensité plus grande et la rapidité d'analyse avantagent la spectroscopie Auger tandis que la simplicité d'exploitation des spectres, la mise en évidence moins ambigüe du glissement chimique, les perspectives d'analyse quantitative plus raffinée et le caractère moins destructif de la méthode privilégient la spectroscopie photoélectronique $\mathrm{X}$.

Cette dernière méthode pourtant présentant l'inconvénient de n'avoir pas un caractère microanalytique et ne permettant donc pas l'obtention d'images, nous 
avons suggéré la conception d'un appareil qui comble ce handicap (vis-à-vis de la spectrométrie Auger); on peut donc désormais espérer atteindre une résolution spatiale de l'ordre de un micron ou mieux (avec, en contrepartie, certaines contraintes supplémentaires - échantillons minces en particulier).

La construction d'appareils permettant l'étude successive du même échantillon par ces deux techniques spectrométriques est donc particulièrement souhaitable et le dispositif que nous préconisons permet d'atteindre ce but sans difficultés. Il suffit en effet d'adjoindre à l'appareil décrit plus haut, un canon à électrons placé sur l'axe de l'analyseur cylindrique (point (9) de la Fig. 1) pour obtenir le signal Auger correspondant à la zone analysée en ESCA.

En outre, comme un objet ayant été le siège de l'effet photoélectronique $X$ se désexcite par fluorescence $X$ et effet Auger et qu'il n'est donc pas rare de trouver des raies Auger dans les spectres ESCA, ce dispositif permettra aussi de combiner, sur le même échantillon, l'analyse Auger induite par des électrons à l'analyse Auger induite par des rayons $\mathrm{X}$.

6. Conclusion générale. - A partir d'une conception originale, nous avons établi qu'il était possible de conférer un caractère microanalytique à la spectroscopie des photoélectrons $X$ puisqu'en utilisant une anticathode en aluminium de $3,3 \mu \mathrm{m}$ d'épaisseur et en l'éclairant avec des électrons de 14,5 keV

$$
\left(I_{0} \simeq 9 \mu \mathrm{A}-d_{0} \simeq 4 \mu \mathrm{m}\right)
$$

$80 \%$ de l'intensité photoélectronique caractéristique sera issue d'une aire circulaire de $7,5 \mu \mathrm{m}$ de rayon.

Par l'emploi d'anticathodes en niobium (voir Tableau IV) on peut aisément atteindre une résolution spatiale inférieure au micron $(80 \%$ de l'intensité étant issue d'une aire circulaire dont le rayon est de l'ordre de $0,5 \mu \mathrm{m}$ ) tout en conservant un signal électronique caractéristique suffisamment intense $\left(10^{4} \mathrm{cps} / \mathrm{s}\right)$ à condition que les conditions optimales soient scrupuleusement respectées (épaisseur de l'anticathode $0,35 \mu \mathrm{m}$ - énergie des électrons incidents : $6 \mathrm{keV}$ $\left.I_{0} \simeq 45 \mathrm{nA}, d_{0} \simeq 0,7 \mu \mathrm{m}\right)$.

Bien que théoriques, nous avons essayé de faire des estimations raisonnables qui ne font pas nécessairement appel aux derniers raffinements techniques aussi avons-nous suggéré des améliorations possibles.

En réduisant ainsi les dimensions de l'aire susceptible d'être analysée par la spectroscopie des photoélectrons $X$, nous supprimons (au prix de quelques inconvénients) certains désavantages que cette technique pouvait présenter vis-à-vis de la spectroscopie Auger et les performances attendues du mode $\grave{a}$ balayage sont comparables à celles de la microscopie Auger à balayage.

Enfin après avoir énuméré les caractères spécifiques de chaque méthode (AES et XPS), nous indiquons comment le dispositif préconisé autorise sur le même échantillon la spectrométrie photoélectronique $\mathrm{X}$, la spectrométrie Auger induite par des rayons $X$ et la spectrométrie Auger induite par des électrons ; sa réalisation nous semble donc présenter un intérêt éminent.

\section{Bibliographie}

[1] Siegbahn, K and coll., Nova Acta Regiae Soc. 20 (1967).

[2] Asilomar, Electron Spectroscopy Inter. Conf. (Shirley Ed.) (1971).

[3] Namur, Electron Spectroscopy: Progress in Research and applications R. Caudano and J. Verbist Editors, J. Electron Spectrosc. Relat. Phenom. 5 (1974).

[4] Cazaux, J., Revue Phys. Appl. 8 (1973) 371.

[5] Ichikawai et Tochigi, M., 7th Int. Cong. Elec. Micr. Grenoble 2. (1970) 117.

[6] Citrin, P. H., Shaw, R. W. et Thomas, T. D., Electron Spectroscopy Asilomar 105 (1972).

[7] Staib, P., Vacuum 22 (1972) 491.

[8] Castaing, R., Adv. Electron. Electron Phys. 13 (1960) 317.

[9] Archard, G., Br. J. Appl. Phys. 32 (1961) 1505.

[10] Philibert, J., Mét. Corros. Ind. 465 (1964) 157 ; 466 (1964) $216 ; 467$ (1964) 325.

[11] Castaing, R. et Deschamps, J., J. Physique Rad. 16 (1955) 304.

[12] Durand, E., Electrostatique (Les distributions Masson Ed.) 1964, 337.

[13] Henke, B., Phys. Rev. 16 (1972) 94.

[14] Fadley, C. S., Namur Conf. J. Electron Spectrosc. and Related Phenom. 5 (1974) 735 et FADLey, C. S. et coll., (1974) J. Electron spectrosc. (à paraître).

[15] Krause, M. O., Chem. Phys. Lett. 10 (1971) 65

[16] Coslett, V. E., Br. J. Appl. Phys. 15 (1964) 107.

[17] Green et Coslett, V. E., Br. J. Appl. Phys. I (1968) 425.

[18] Loeffler, K. M., 7th Int. Cong. Elec. Micr. Grenoble 2. (1970) $77,33$.
[19] Wuillemier, F. et Krause, M. O., Electron spectroscopy Asilomar (1972) 259.

[20] Kemeny, P. C. et coll., Phys. Rev. B 9 (1974) 5307.

[21] Veigele, W. M. J., X-Ray cross section compilation Kaman Sciences CORP Report No 24 113F (1971).

[22] Smithell, C. H., Metal Reference Book III (1967) 685.

[23] MulveY, T., Focusing of charged particles Septier Ed. I (1967) 469

[24] Veneklassen, L. M., Optik 36 (1972) 410.

[25] MacDonald, N. C. et Waldrop, J. R., Appl. Phys. Lett. 19 (1971) 315.

[26] Griffit, HS. B. et WARdell, I. R. M., Scanning Electron Microscopy (Newcastle) 18 (1973) 42.

[27] Ebel, M. F., Namur Conf. J. Elect. Spectrosc. 5 (1974) 837.

[28] BAUER, E., Vacuum 22 (1972) 539.

[29] Todd, C. J., Vacuum 23 (1973) 195.

[30] Brundle, C. R., J. Vac. Sci. Technol. 11 (1974) 212

[31] Poweli, C. J., Surf. Sci. 44 (1974) 29.

[32] Chang, C. C., Surf. Sci. 25 (1971) 53.

[33] Broers, A. N., Critical review in solid state sciences (1974) 333.

[34] Booker, G. R., Modern diffraction and imaging techniques in material science, $\mathrm{S}$. Amelinckx and al. (North Holland) 1970 p. 553.

[35] CoAd, J. P., J. Electron. Spectrosc. and Relat. Phenom. (à paraître) citée par PIERCE, D. T. Acta electron. 18 (1975) 69. 